Article

\title{
On the Tautomerism of $N$-Substituted Pyrazolones: 1,2-Dihydro-3H-pyrazol-3-ones versus 1H-Pyrazol-3-ols
}

\author{
Eglè Arbačiauskienè ${ }^{1}$, Sonata Krikštolaityte ${ }^{1}$, Aiva Mitrulevičienè ${ }^{1}$, Aurimas Bieliauskas ${ }^{2}$, \\ Vytas Martynaitis ${ }^{1}$, Matthias Bechmann ${ }^{3}$ (D), Alexander Roller ${ }^{4}$, Algirdas Šačkus ${ }^{1,2, *}$ \\ and Wolfgang Holzer ${ }^{5, *}$ \\ 1 Department of Organic Chemistry, Kaunas University of Technology, Radvilènu pl. 19, \\ LT-50254 Kaunas, Lithuania; egle.arbaciauskiene@ktu.lt (E.A.); sonata.krikstolaityte@ktu.lt (S.K.); \\ aiva.mitruleviciene@gmail.com (A.M.); vytas.martynaitis@ktu.lt (V.M.) \\ 2 Institute of Synthetic Chemistry, Kaunas University of Technology, K. Baršausko g. 59, \\ LT-51423 Kaunas, Lithuania; aurimas.bieliauskas@ktu.lt \\ 3 Institute of Organic Chemistry, Johannes Kepler University Linz, Altenberger Straße 69, \\ A-4040 Linz, Austria; matthias.bechmann@jku.at \\ 4 X-ray Structure Analysis Centre, Faculty of Chemistry, University of Vienna, Währinger Straße 42, \\ A-1090 Vienna, Austria; alexander.roller@univie.ac.at \\ 5 Department of Pharmaceutical Chemistry, Faculty of Life Sciences, University of Vienna, Althanstrasse 14, \\ A-1090 Vienna, Austria \\ * Correspondence: algirdas.sackus@ktu.lt (A.Š.); wolfgang.holzer@univie.ac.at (W.H.); \\ Tel.: +370-37-451401 (A.Š.); +43-1-4277-55623 (W.H.)
}

Received: 27 November 2017; Accepted: 30 December 2017; Published: 9 January 2018

\begin{abstract}
The tautomerism of 1-phenyl-1,2-dihydro-3H-pyrazol-3-one was investigated. An X-ray crystal structure analysis exhibits dimers of 1-phenyl-1H-pyrazol-3-ol units. Comparison of NMR (nuclear magnetic resonance) spectra in liquid state $\left({ }^{1} \mathrm{H},{ }^{13} \mathrm{C},{ }^{15} \mathrm{~N}\right)$ with those of "fixed" derivatives, as well as with the corresponding solid state NMR spectra reveal this compound to exist predominantly as $1 H$-pyrazol-3-ol molecule pairs in nonpolar solvents like $\mathrm{CDCl}_{3}$ or $\mathrm{C}_{6} \mathrm{D}_{6}$, whereas in DMSO- $d_{6}$ the corresponding monomers are at hand. Moreover, the NMR data of different related $1 \mathrm{H}$-pyrazol-3-ol derivatives are presented.
\end{abstract}

Keywords: prototropic tautomerism; $1 H$-pyrazol-3-ol; 1,2-dihydro-3H-pyrazol-3-ones; NMR $\left({ }^{1} \mathrm{H}\right.$; ${ }^{13} \mathrm{C} ;{ }^{15} \mathrm{~N}$ ); solid state NMR; X-ray structure analysis

\section{Introduction}

Pyrazolones are interesting chemical entities not only due to their importance as building blocks for the synthesis of various bio-active compounds [1-6], but also in respect to their capability to prototropic tautomerism and to the-more uncommon-phenomenon of desmotropy [7]. Whereas for 1-substituted $1 \mathrm{H}$-pyrazol-5-ols and their tautomers (2-substituted 2,4-dihydro-3H-pyrazol-3-ones and 2-substituted 1,2-dihydro-3H-pyrazol-3-ones), a considerably large number of experimental and theoretical studies concerning their tautomerism has been published [8-12], there is much less known about their structural isomers with a 1-substituted $1 \mathrm{H}$-pyrazol-3-ol motif. The latter compounds are in as much of interest as they can serve as starting materials for further functionalization [13], the construction of anellated systems [14], as well as for the synthesis of biologically active compounds [15-17]. Hence, this study is devoted to investigations with 1-substituted $1 \mathrm{H}$-pyrazol-3-ols (tautomers to the corresponding 1,2-dihydro-3H-pyrazol-3-ones) and some derivatives carrying different substituents at pyrazole C-4. 


\section{Results and Discussion}

In principle, for the title compounds, two tautomeric forms are possible, namely the OH-form and the NH-form (Figure 1). In Chemical Abstracts such compounds carrying an alkyl or a (hetero)aryl substituent at the pyrazole nitrogen atom are always listed as $3 \mathrm{H}$-pyrazol-3-ones. Hence, as many authors prefer the latter denomination, in the course of this study we investigated which tautomer is really relevant in a solid state and, especially, in solution.

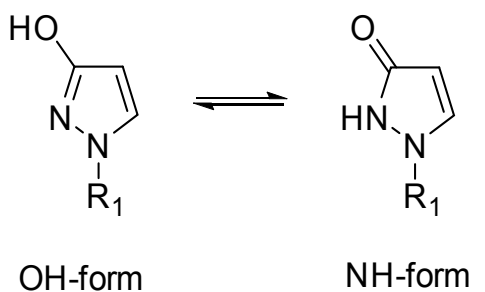

Figure 1. Possible tautomeric forms of 1-substituted 3-hydroxy-1H-pyrazoles (1-substituted 1,2-dihydro3H-pyrazol-3-ones).

\subsection{X-ray Analysis of 1-Phenyl-1,2-dihydro-3H-pyrazol-3-one (1-Phenyl-1H-pyrazol-3-ol) (1)}

In the solid state an unambiguous determination of structure and, thus, a safe discrimination between $\mathrm{OH}$ and $\mathrm{NH}$-form is possible. In view of this fact crystals of 1-phenyl-1,2-dihydro-3Hpyrazol-3-one (1-phenyl-1H-pyrazol-3-ol) (1)_obtained by crystallization from ethanol/water-were subjected to X-ray structure analysis. It turned out that this compound is present in the $1 H$-pyrazol-3-ol form constituting dimeric units connected by two identical intermolecular hydrogen bonds (Figure 2). In principle, the formation of similar dimeric structures would be also possible by combination of two identical $\mathrm{NH}$-isomers establishing two intermolecular hydrogen bonds between $\mathrm{C}=\mathrm{O}$ and the $\mathrm{NH}$ of the second molecule. However, the electron density map clearly shows the position of the hydrogen atom at the oxygen and thus excludes the latter alternative (Figure 2, further details can be found in the Experimental Section).

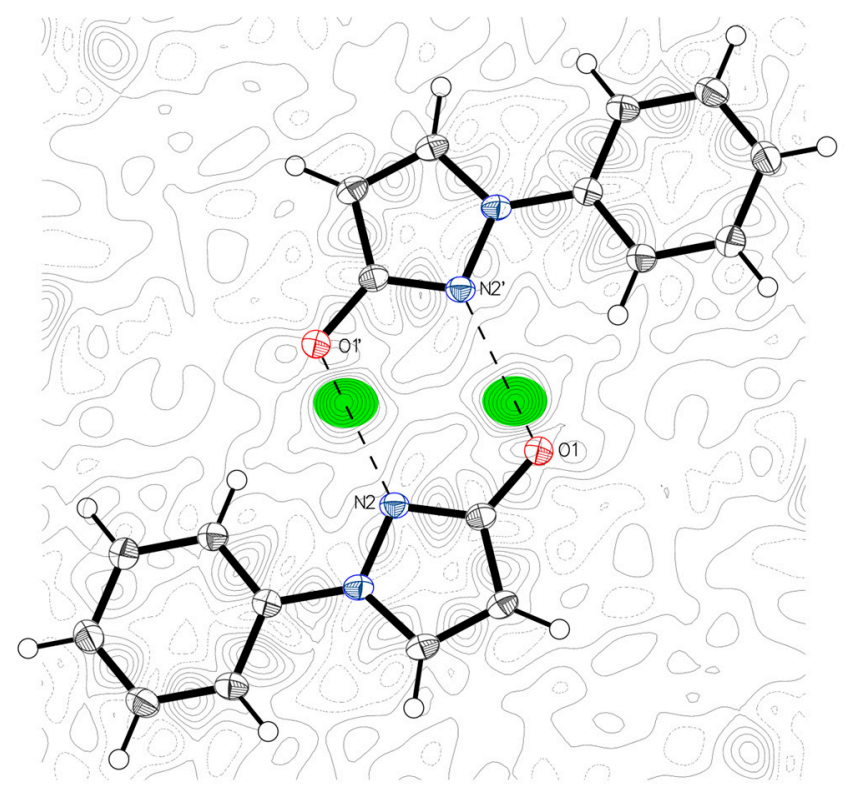

Figure 2. Difference electron density map of $\mathbf{1}$. Determination of the $\mathrm{H}$ position at $\mathrm{O} 1$. The distance of O1 to the electron density position (green shaded) in the direct donor acceptor line is 0.8927 (10) A. The according distance to N2' is 1.8339 (10) A. For further details please follow the CCDC Code. 


\subsection{Solid State NMR (SSNMR) of $\mathbf{1}$}

The same material as used for the X-ray analysis was subjected to solid state NMR (CP/MAS). As the X-ray analysis revealed the presence of the $1 H$-pyrazol-3-ol form, the solid state NMR spectra also should exclusively origin from this species. Here, due to its simplicity the ${ }^{15} \mathrm{~N}-\mathrm{NMR}$ spectrum is particularly valuable, showing the "pyridine-like" pyrazole N-atom (N-2) at 243.1 ppm, whereas the "pyrrole-like" N-atom (N-1) is resonating at 192.6 ppm referenced against external ${ }^{15} \mathrm{NH}_{4} \mathrm{Cl}(39.3 \mathrm{ppm}$ with respect to liquid $\mathrm{NH}_{3}$ ) (Figure 3). The distinct chemical shift difference of N-1 compared to N-2 $(\Delta \delta=50.5 \mathrm{ppm})$ clearly reflects the fact that the two nitrogen atoms are of different types.

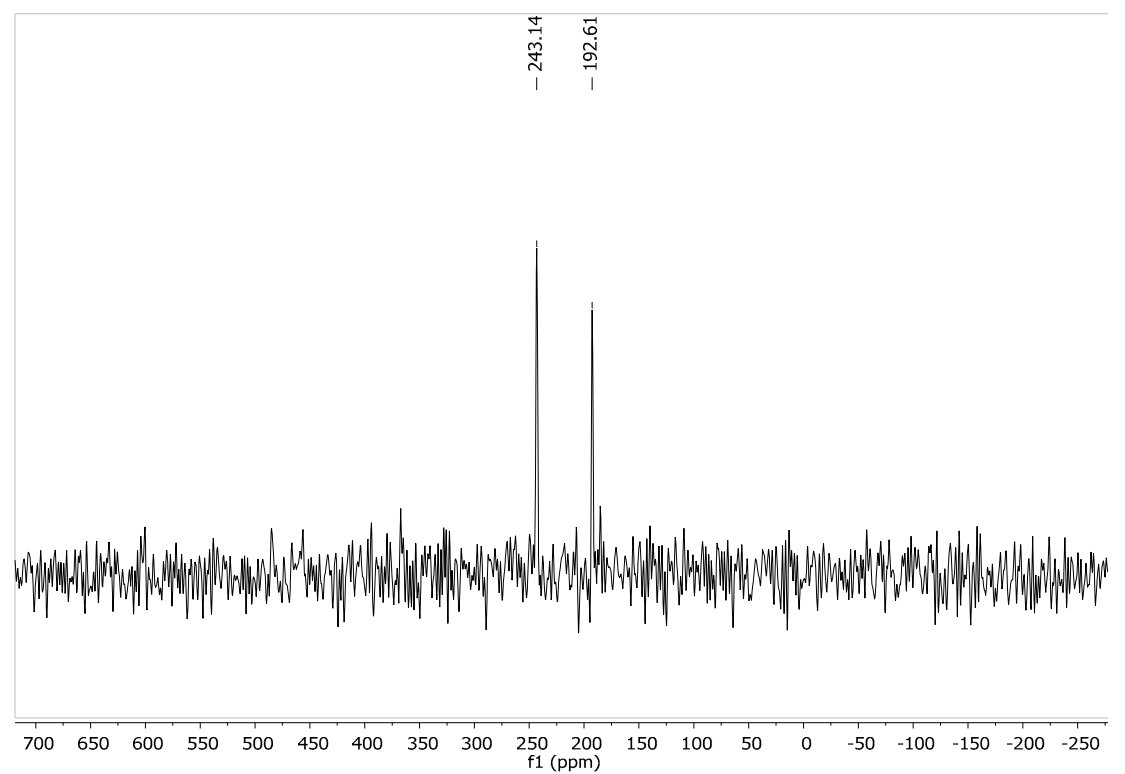

Figure $3 .{ }^{15} \mathrm{~N} \mathrm{CP} / \mathrm{MAS}$ NMR spectrum of $\mathbf{1}$.

In addition, in ${ }^{15} \mathrm{~N}-\mathrm{NQS}$ (non-quaternary suppression) experiments with different pre-scan dephasing delays (20 $\mu \mathrm{s}, 100 \mu \mathrm{s}, 200 \mu \mathrm{s})$ the intensities of both ${ }^{15} \mathrm{~N}-\mathrm{NMR}$ resonances remained constant. This behaviour suggests that the two nitrogen atoms are of the same type regarding their protonation status what is only the case for the $\mathrm{OH}$ isomer.

\subsection{NMR Spectra in Solution}

Whereas in the solid state, an unambiguous determination of individual tautomeric forms is smoothly possible by X-ray structure analysis, the situation in solution is much more complex. Here, depending on a plethora of different influencing variables, tautomeric equilibria with the simultaneous presence of several tautomeric forms are possible, whereas time-averaged signals are obtained in case of fast exchange. Amongst the appropriate methods for investigating such tautomeric equilibria in solution NMR spectroscopic methods play a prominent role [8,9,18-20]. A frequently used concept is the comparison of the data obtained in solution with those of "fixed" derivatives (representing the individual "frozen" tautomeric forms) or with the data of the individual tautomeric forms obtained from solid state NMR experiments. Although this approach comes along with some difficulties (i.e. estimating the difference between the tautomer and the model compound) in many cases fairly good results can be obtained, particularly when one tautomeric form is strongly dominating. However, in cases when several forms are present to a significant extent the precise determination of the percentage composition by interpolation is difficult.

As compound $\mathbf{1}$ is present as 1 H-pyrazol-3-ol in the solid state, a comparison of the crucial SSNMR chemical shifts with those in solution should provide valuable information. As outlined in Figure 4, the ${ }^{13} \mathrm{C}$ and the ${ }^{15} \mathrm{~N}$ chemical shifts at the pyrazole nucleus show a high degree of accordance between 
the solid state and those in $\mathrm{CDCl}_{3}$ or in $\mathrm{C}_{6} \mathrm{D}_{6}$ solution which leads to the conclusion that the 3-hydroxy form is far dominating in these solvents.

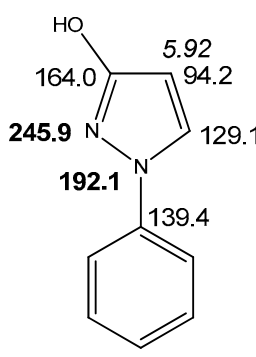

$1\left(\mathrm{CDCl}_{3}\right)$

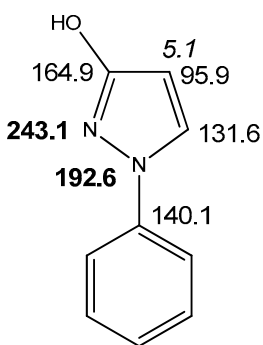

1 (solid state)

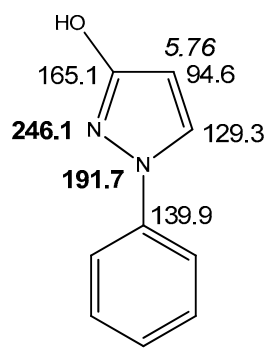

$1\left(\mathrm{C}_{6} \mathrm{D}_{6}\right)$

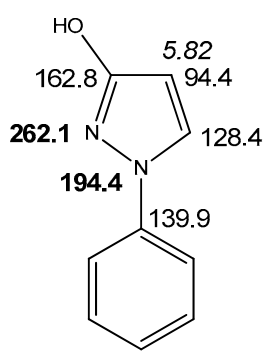

$1\left(\mathrm{DMSO}-d_{6}\right)$

Figure 4. Crucial chemical shifts of 1 in solid state and in different solvents. ${ }^{1} \mathrm{H}-\mathrm{NMR}$ chemical shifts are represented in italics, ${ }^{13} \mathrm{C}-\mathrm{NMR}$ chemical shifts in plain text, ${ }^{15} \mathrm{~N}-\mathrm{NMR}$ chemical shifts in bold.

In DMSO- $d_{6}$ solution it is noticeable that the signal of pyrazole N-2 is clearly shifted downfield compared to the recordings in $\mathrm{CDCl}_{3}$ or $\mathrm{C}_{6} \mathrm{D}_{6}$. A possible explanation for this phenomenon is the fact that in the latter nonpolar solvents $\mathbf{1}$ is obviously present as a dimer of $1 H$-pyrazol-3-ols (like in the solid state) and, thus, the pyrazole N-2 atom is involved in an intramolecular hydrogen bond, whereas-in contrast - in the strong acceptor solvent DMSO- $d_{6}$ these intermolecular hydrogen bonds are broken and now monomers are dominating. It is well-known that involvement of a nitrogen's lone-pair in hydrogen bonds (or-to a larger extent-oxidation, alkylation, or complexation) leads to a marked upfield shift of the corresponding ${ }^{15} \mathrm{~N}$ resonance [21-23]. In 3-methoxy-1-phenyl-1H-pyrazole (2) (Figure 5) the above mentioned dimerization and, thus, participation of pyrazole N-2 into intermolecular hydrogen bonding is not possible, what is reflected by a larger chemical shift of the latter. Hence, $\delta(\mathrm{N}-2)(261.7 \mathrm{ppm})$ is now comparable to the value in DMSO- $d_{6}(262.1 \mathrm{ppm})$, whereas $\delta(\mathrm{N}-1)$ in compound 2 (195.6 ppm) and in $\mathbf{1}$ in different solvents (191.7-194.5 ppm) is very similar. Hence, it can be concluded that $\mathbf{1}$ is also present as $1 H$-pyrazol-3-ol in DMSO- $d_{6}$ solution, however, not in the dimeric form stabilized by intermolecular hydrogen bonds.

In addition, employing the concept of the fixed derivatives we compared the ${ }^{1} \mathrm{H}-,{ }^{13} \mathrm{C}$ - and ${ }^{15} \mathrm{~N}-\mathrm{NMR}$ chemical shifts, as well as characteristic spin coupling constants of 1 with its $O$-methyl (2) and N-methyl derivative (3), the "fixed" OH- and NH-forms, respectively.

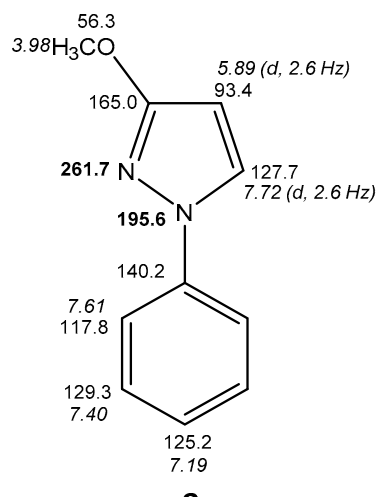

2

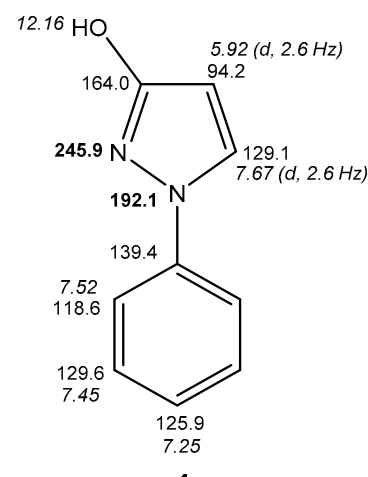

1

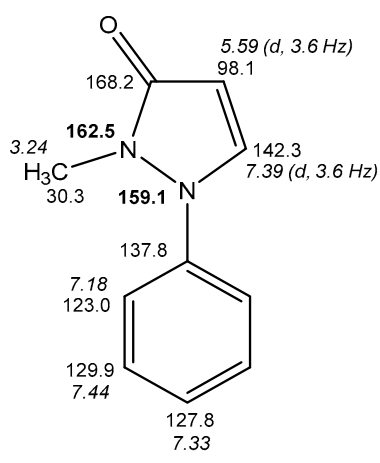

3

Figure 5. ${ }^{1} \mathrm{H}-\mathrm{NMR}$ (in italics), ${ }^{13} \mathrm{C}-\mathrm{NMR}$, and ${ }^{15} \mathrm{~N}-\mathrm{NMR}$ (in bold) chemical shifts of $\mathbf{1}$ and its "fixed" derivatives 2 and 3 (in $\mathrm{CDCl}_{3}$ ).

From the data given in Figure 5 the above conclusions are confirmed, namely that compound 1, which in principle is capable of prototropic tautomerism, is predominantly existing as $\mathrm{OH}$-isomer in $\mathrm{CDCl}_{3}$ solution. This is supported by the fact that the ${ }^{1} \mathrm{H}-,{ }^{13} \mathrm{C}-$, and ${ }^{15} \mathrm{~N}-\mathrm{NMR}$ chemical shifts 
of $\mathbf{1}$ resemble closely to those of the fixed O-methyl congener 2. Especially the ${ }^{15} \mathrm{~N}$-chemical shifts are valuable indicators, as the $\mathrm{N}$-methyl derivative 3 exhibits two $\mathrm{sp}^{3}$-type nitrogen atoms with similar ${ }^{15} \mathrm{~N}-\mathrm{NMR}$ chemical shifts, whereas in $\mathbf{1}$ and $\mathbf{2}$ the large chemical shift differences between the two nitrogen atoms in the corresponding molecule hint to different types of $\mathrm{N}$-atoms $\left(\mathrm{sp}^{3}\right.$ and $\mathrm{sp}^{2}$ ). For comparison only, with 1-phenylpyrazolidin-3-one, which formally is the dihydro derivative of the NH form of $\mathbf{1}$, we found $105.1 \mathrm{ppm}$ for N-1 and $151.8 \mathrm{ppm}$ for N-2 in DMSO- $d_{6}$ solution. Moreover, $\mathbf{1}$ and $\mathbf{2}$ show equal sizes of the vicinal ${ }^{3} \mathrm{~J}(\mathrm{H} 4, \mathrm{H} 5)$ coupling constant at the pyrazole nucleus $(2.6 \mathrm{~Hz})$, whereas in 3 this coupling is considerably larger $(3.6 \mathrm{~Hz})$ (Figure 5). An additional difference consists in the ${ }^{13} \mathrm{C}-\mathrm{NMR}$ chemical shift of Ph C-2/6 which is akin in $\mathbf{1}(118.6 \mathrm{ppm})$ and $\mathbf{2}$ (117.8 ppm). In contrast, 3 shows a markedly larger chemical shift for these carbon atoms (123.0 ppm) which can be attributed to some distorsion of phenyl and pyrazole ring obviously induced by the sterical hindrance of the N-methyl group [24,25]. Additionally, the large differences in ${ }^{13} \mathrm{C}-\mathrm{NMR}$ chemical shifts of pyrazole C-5 (1: $129.1 \mathrm{ppm}, 2: 127.7 \mathrm{ppm})$ in comparison to that of 3 (142.3 ppm) provide an extra confirmation.

Moreover, ${ }^{1} \mathrm{H}-,{ }^{13} \mathrm{C}-$, and ${ }^{15} \mathrm{~N}-\mathrm{NMR}$ spectra of compound $\mathbf{1}$ were additionally taken from $\mathrm{C}_{6} \mathrm{D}_{6}$, DMSO- $d_{6}$ and $\mathrm{CD}_{3}$ OD solutions. As all the significant criteria discussed above were almost similar to those in $\mathrm{CDCl}_{3}$ solution, it is reasoned that, also in these solvents, the hydroxy form is far more predominant. The regarding data are presented in the Experimental Section.

In the following, congeners of $\mathbf{1}$ carrying a halogen atom or an acyl moiety at pyrazole C-4 were investigated (compounds 4-8). Again, all these species clearly exist as pyrazol-3-ols in $\mathrm{CDCl}_{3}$, as well as in DMSO- $d_{6}$ solution based on the ${ }^{13} \mathrm{C}$ - and ${ }^{15} \mathrm{~N}-\mathrm{NMR}$ chemical shift considerations outlined above. Regarding the 4-bromo derivative 5 a "fixed" 3-methoxy derivative 9 has been described already by us, whose data resemble the "free" $1 H$-pyrazol-3-ol 5 [26]. The same is the case for the pair $\mathbf{7}$ and $\mathbf{1 0}$ (Figure 6). When switching from $\mathrm{CDCl}_{3}$ to DMSO- $d_{6}$ solution, the ${ }^{13} \mathrm{C}$ chemical shift of the carbonyl C-atom in 7 receives an upfield shift of $4.0 \mathrm{ppm}(195.7 \mathrm{ppm} \rightarrow 191.7 \mathrm{ppm})$ which hints to the existence of an intramolecular hydrogen bond-but now-between carbonyl O-atom and $\mathrm{OH}$ proton in $\mathrm{CDCl}_{3}$ solution, which is broken in the strong acceptor solvent DMSO- $d_{6}$ [27]. In principle, also 3-O-acyl derivatives of $\mathbf{1}$ (compounds 11-13) can be regarded as fixed 3-OH derivatives, although the 3-O-acyl rest seems to be less comparable to $\mathrm{OH}$ than an $\mathrm{OCH}_{3}$ group. However, despite larger differences regarding the ${ }^{13} \mathrm{C}$ chemical shifts of the pyrazole $\mathrm{C}$-atoms between $\mathbf{1}$ and 11-13 appear, the data of the phenyl ring closely resemble as well as the ${ }^{3} \mathrm{~J}(\mathrm{H} 4, \mathrm{H} 5)$ coupling constant at the pyrazole nucleus, which for $\mathbf{1 1 - 1 3}$ is the same as in $\mathbf{1}(2.5-2.6 \mathrm{~Hz})$.

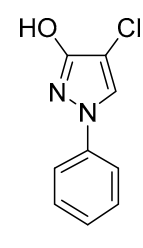

4

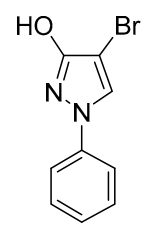

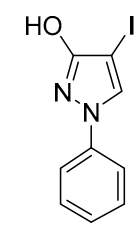

6

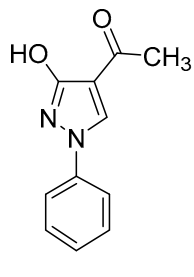

7

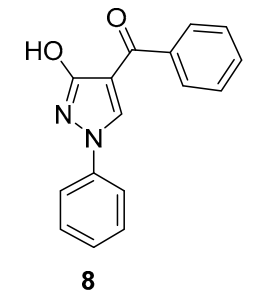

8

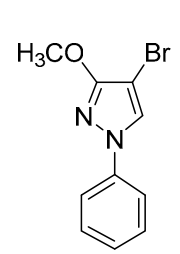

9

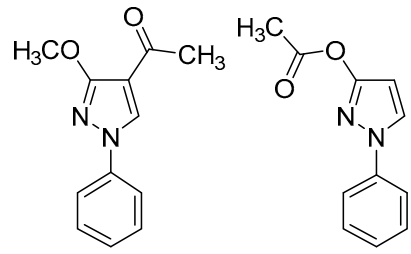

10

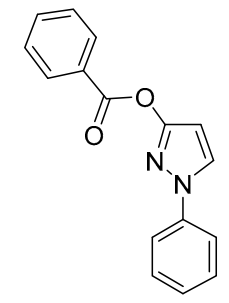

12

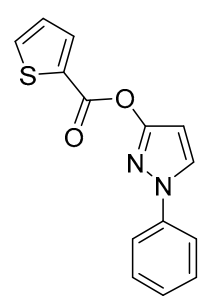

13

Figure 6. Investigated 4-substituted 1-phenyl-1H-pyrazol-3-ols (4-8) and 3-methoxy congeners $(\mathbf{9}, 10)$, as well as 3-O-acyl derivatives of $\mathbf{1}$ (11-13). 
The triple 14-16 provides another example of comparing a free $1 H$-pyrazol-3-ol (14) with the corresponding $O$-alkyl (15) and $N$-alkyl derivative (16), respectively. Again, the selected data depicted in Figure 7 clearly hint that $\mathbf{1 4}$ predominantly exists as $\mathrm{OH}$-isomer and not as pyrazol-3-one.

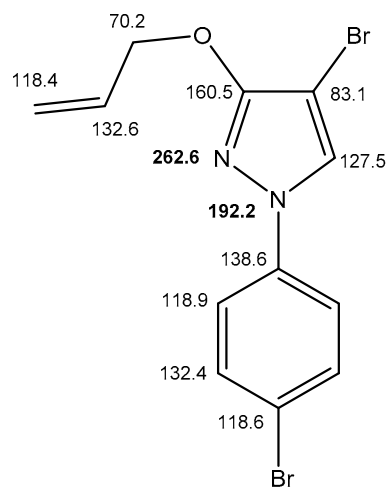

15

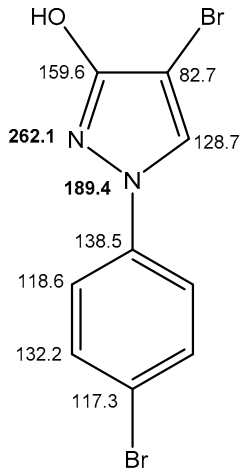

14

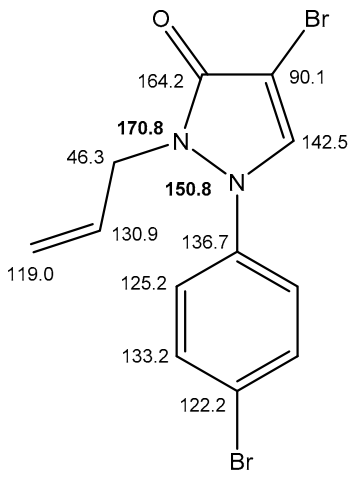

16

Figure 7. ${ }^{1} \mathrm{H}$ (in italics), ${ }^{13} \mathrm{C}$ and ${ }^{15} \mathrm{~N}$ (in bold) NMR chemical shifts of $\mathbf{1 4}$ and its "fixed" derivatives $\mathbf{1 5}$ and 16 (in $\mathrm{CDCl}_{3}$ ).

In addition, we investigated $1 H$-pyrazol-3-ols carrying a methyl (17) and a benzyl substituent (18), respectively, at pyrazole N-1. From the relevant data of these compounds, depicted in Figure 8, the conclusion can be drawn, that also 17 and 18 exist in the 3-hydroxy form in $\mathrm{CDCl}_{3}, \mathrm{DMSO}-d_{6}$, and $\mathrm{C}_{6} \mathrm{D}_{6}$ solution. Again, as found with 1-phenyl- $1 \mathrm{H}$-pyrazol-3-ol 1 and compounds 17, 18, the markedly larger chemical shifts of pyrazole N-2 in DMSO- $d_{6}$ compared to those in $\mathrm{CDCl}_{3}$ or $\mathrm{C}_{6} \mathrm{D}_{6}$ hint to the absence of dimers stabilized by intermolecular hydrogen bonds in this solvent, what is supported by a distinctly smaller ${ }^{1} \mathrm{H}-\mathrm{NMR}$ chemical shift of the OH-proton in DMSO- $d_{6}$ (Figure 8).

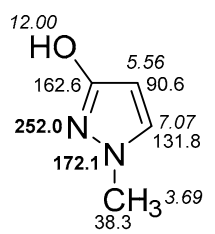

$17\left(\mathrm{CDCl}_{3}\right)$

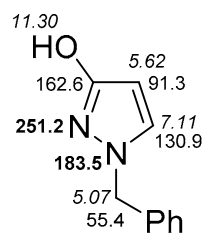

$18\left(\mathrm{CDCl}_{3}\right)$

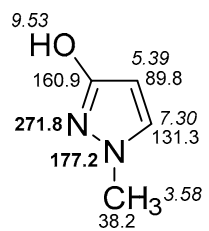

$17\left(\right.$ DMSO- $\left.d_{6}\right)$

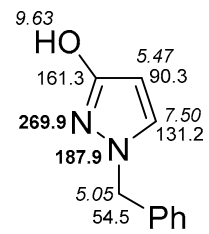

$18\left(\mathrm{DMSO}-d_{6}\right)$

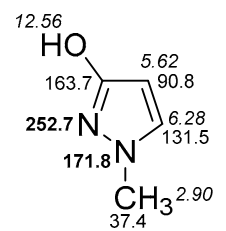

$17\left(\mathrm{C}_{6} \mathrm{D}_{6}\right)$

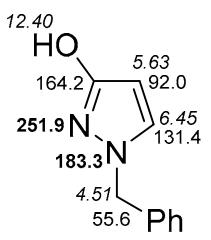

$18\left(\mathrm{C}_{6} \mathrm{D}_{6}\right)$

Figure 8. Crucial ${ }^{1} \mathrm{H}$ (in italics), ${ }^{13} \mathrm{C}$, and ${ }^{15} \mathrm{~N}$ (in bold) NMR chemical shifts of 1-methyl-1H-pyrazol-3-ol (17) and 1-benzyl-1H-pyrazol-3-ol (18) in $\mathrm{CDCl}_{3}$, DMSO- $d_{6}$, and $\mathrm{C}_{6} \mathrm{D}_{6}$ solution.

\section{Experimental Section}

\subsection{General Information}

Melting points were determined on a Büchi M-565 melting point apparatus (Büchi Labortechnik AG, Flawil, Switzerland) and are uncorrected. IR (infrared) spectra ( $\mathrm{KBr}$ pellets) were recorded on 
a Bruker Tensor 27 spectrometer (Bruker Optik GmbH, Ettlingen, Germany) and are reported in wave numbers $\left(\mathrm{cm}^{-1}\right)$. High-resolution ESI-TOF mass spectra were measured on a Bruker maXis spectrometer (Bruker Daltonik GmbH, Bremen, Germany). Elemental analyses were performed at the Microanalytical Laboratory, University of Vienna. ${ }^{1} \mathrm{H}$ and ${ }^{13} \mathrm{C}-\mathrm{NMR}$ spectra were recorded on a Bruker Avance 500 spectrometer (500.13 MHz for ${ }^{1} \mathrm{H}, 125.77 \mathrm{MHz}$ for ${ }^{13} \mathrm{C}$ ) (Bruker BioSpin $\mathrm{GmbH}$, Rheinstetten, Germany-valid for all mentioned Bruker NMR spectrometers), a Bruker Avance III 400 spectrometer $\left(400.23 \mathrm{MHz}\right.$ for ${ }^{1} \mathrm{H}, 100.64 \mathrm{MHz}$ for $\left.{ }^{13} \mathrm{C}\right)$ or a Varian UnityPlus300 spectrometer (299.95 MHz for ${ }^{1} \mathrm{H}, 75.43 \mathrm{MHz}$ for ${ }^{13} \mathrm{C}$ ) (Varian, Palo Alto, CA, USA) at $293 \mathrm{~K}$. The centre of the solvent signal was used as an internal standard which was related to TMS with $\delta 7.26 \mathrm{ppm}\left({ }^{1} \mathrm{H}\right.$ in $\left.\mathrm{CDCl}_{3}\right)$, $\delta 2.49 \mathrm{ppm}\left({ }^{1} \mathrm{H}\right.$ in DMSO- $\left.d_{6}\right), \delta 7.16 \mathrm{ppm}\left({ }^{1} \mathrm{H}\right.$ in $\left.\mathrm{C}_{6} \mathrm{D}_{6}\right), \delta 3.31 \mathrm{ppm}\left({ }^{1} \mathrm{H}\right.$ in $\left.\mathrm{CD}_{3} \mathrm{OD}\right), \delta\left(\delta 77.0 \mathrm{ppm}\left({ }^{13} \mathrm{C}\right.\right.$ in $\left.\mathrm{CDCl}_{3}\right), \delta 39.5 \mathrm{ppm}\left({ }^{13} \mathrm{C}\right.$ in DMSO- $\left.d_{6}\right), \delta 128.06 \mathrm{ppm}\left({ }^{13} \mathrm{C}\right.$ in $\left.\mathrm{C}_{6} \mathrm{D}_{6}\right)$ and $\delta 49.00 \mathrm{ppm}\left({ }^{13} \mathrm{C}\right.$ in CD $\left.\mathrm{OD}\right)$. The digital resolutions were $0.20 \mathrm{~Hz}$ /data point in the ${ }^{1} \mathrm{H}$ and $0.33 \mathrm{~Hz} /$ data point in the ${ }^{13} \mathrm{C}-\mathrm{NMR}$ spectra. ${ }^{15} \mathrm{~N}-\mathrm{NMR}$ spectra were obtained on Bruker Avance 500 (50.69 MHz) and Bruker Avance III 400 (40.56 MHz) spectrometers (both equipped with "direct" detection broadband z-gradient observe probes) or on a Bruker Avance III $700(70.96 \mathrm{MHz})$ equipped with a $5 \mathrm{~mm} \mathrm{TCI}{ }^{1} \mathrm{H}_{-}{ }^{13} \mathrm{C} /{ }^{15} \mathrm{~N} / \mathrm{D}$ z-gradient cryoprobe, and were measured against external nitromethane (coaxial capillary) and recalculated to liquid ammonia. Solid-state NMR spectra (CP/MAS, MAS: $10 \mathrm{kHz}$ ) were recorded on a Bruker Avance III 500 instrument with a broadband MAS-probe for $3.2 \mathrm{~mm}$ rotors. CP contact times were $2 \mathrm{~ms}$ for $\left({ }^{1} \mathrm{H},{ }^{13} \mathrm{C}\right)$ and $3 \mathrm{~ms}$ for $\left({ }^{1} \mathrm{H},{ }^{15} \mathrm{~N}\right) .{ }^{1} \mathrm{H}$ RF of $100 \mathrm{kHz}$ was used for spinal64 broadband decoupling. ${ }^{1} \mathrm{H},{ }^{13} \mathrm{C}$-HETCOR spectra were recorded using FSLG homonuclear decoupling during $\mathrm{t}_{1}$-evolution and mixing times of $50 \mu \mathrm{s}$ and $200 \mu \mathrm{s} .{ }^{15} \mathrm{~N}-\mathrm{NMR}$ spectra were referenced to ${ }^{15} \mathrm{NH}_{4} \mathrm{Cl}$ and recalculated to the liquid ammonia scale $\left({ }^{15} \mathrm{NH}_{4} \mathrm{Cl} 39.3 \mathrm{ppm}\right) .{ }^{13} \mathrm{C}$ spectra were referenced to the methylene carbon signals of adamantane and recalculated to the TMS scale $\left(\delta^{13} \mathrm{CH}_{2} 38.5 \mathrm{ppm}\right) .{ }^{1} \mathrm{H}$ chemical shifts were referenced to the $\mathrm{NH}_{3}{ }^{+}$resonance in $\alpha$-Glycine and recalculated to the TMS scale $\left(\delta^{15} \mathrm{NH}_{3}{ }^{+} 8.5 \mathrm{ppm}\right)$. Product yields were not optimised.

\subsection{Data of Investigated Compounds}

1-Phenyl-1H-pyrazol-3-ol (1). Compound 1 was prepared by oxidation of 1-phenylpyrazolidin-3-one with $\mathrm{FeCl}_{3}$ according to Reference [28] and recrystallized from EtOH-H${ }_{2} \mathrm{O} .{ }^{1} \mathrm{H}-\mathrm{NMR}\left(\mathrm{CDCl}_{3}\right): \delta 12.16$ (br s, 1H, OH), $7.67\left(\mathrm{~d},{ }^{3} \mathrm{~J}=2.6 \mathrm{~Hz}, 1 \mathrm{H}\right.$, pyrazole H-5), $7.52(\mathrm{~m}, 2 \mathrm{H}, \mathrm{Ph} \mathrm{H}-2,6), 7.45$ (m, 2H, Ph H-3,5), 7.25 $(\mathrm{m}, 1 \mathrm{H}, \mathrm{Ph} \mathrm{H}-4), 5.92\left(\mathrm{~d},{ }^{3} \mathrm{~J}=2.6 \mathrm{~Hz}, 1 \mathrm{H}\right.$, pyrazole H-4). ${ }^{13} \mathrm{C}-\mathrm{NMR}\left(\mathrm{CDCl}_{3}\right): \delta 164.0\left({ }^{2} J(\mathrm{C} 3, \mathrm{H} 4)=2.2 \mathrm{~Hz}\right.$, ${ }^{3} J(\mathrm{C} 3, \mathrm{H} 5)=10.4 \mathrm{~Hz}$, pyrazole C-3), 139.4 (Ph C-1), $129.6(\mathrm{Ph} \mathrm{C}-3,5), 129.1\left({ }^{1} J(\mathrm{C} 5, \mathrm{H} 5)=187.0 \mathrm{~Hz}\right.$, ${ }^{2} J(\mathrm{C} 5, \mathrm{H} 4)=8.4 \mathrm{~Hz}$, pyrazole C-5), $125.9(\mathrm{Ph} \mathrm{C}-4), 118.6(\mathrm{Ph} \mathrm{C}-2,6), 94.2\left({ }^{1} \mathrm{~J}(\mathrm{C} 4, \mathrm{H} 4)=180.2 \mathrm{~Hz}\right.$, ${ }^{2} J(\mathrm{C} 4, \mathrm{H} 5)=7.7 \mathrm{~Hz}$, pyrazole C-4). ${ }^{15} \mathrm{~N}-\mathrm{NMR}\left(\mathrm{CDCl}_{3}\right): \delta 192.1$ (pyrazole N-1), 245.9 (pyrazole N-2). ${ }^{1} \mathrm{H}-\mathrm{NMR}\left(\mathrm{DMSO}-d_{6}\right): \delta 10.28(\mathrm{~s}, 1 \mathrm{H}, \mathrm{OH}), 8.18\left(\mathrm{~d},{ }^{3} \mathrm{~J}=2.6 \mathrm{~Hz}, 1 \mathrm{H}\right.$, pyrazole H-5), $7.67(\mathrm{~m}, 2 \mathrm{H}$, Ph H-2,6), 7.40 (m, 2H, Ph H-3,5), 7.15 (m, 1H, Ph H-4), 5.82 (d, ${ }^{3} J=2.6 \mathrm{~Hz}, 1 \mathrm{H}$, pyrazole H-4). ${ }^{13} \mathrm{C}-\mathrm{NMR}\left(\mathrm{DMSO}-d_{6}\right): \delta 162.8\left({ }^{2} \mathrm{~J}(\mathrm{C} 3, \mathrm{H} 4)=2.5 \mathrm{~Hz},{ }^{3} \mathrm{~J}(\mathrm{C} 3, \mathrm{H} 5)=10.3 \mathrm{~Hz}\right.$, pyrazole C-3), $139.9(\mathrm{Ph} \mathrm{C}-1)$, $129.4(\mathrm{Ph} \mathrm{C}-3,5), 128.4\left({ }^{1} \mathrm{~J}(\mathrm{C} 5, \mathrm{H} 5)=188.2 \mathrm{~Hz},{ }^{2} \mathrm{~J}(\mathrm{C} 5, \mathrm{H} 4)=8.9 \mathrm{~Hz}\right.$, pyrazole C-5), $124.7(\mathrm{Ph} \mathrm{C}-4), 116.8(\mathrm{Ph}$ $\mathrm{C}-2,6), 94.4\left({ }^{1} \mathrm{~J}(\mathrm{C} 4, \mathrm{H} 4)=178.0 \mathrm{~Hz},{ }^{2} \mathrm{~J}(\mathrm{C} 4, \mathrm{H} 5)=8.0 \mathrm{~Hz}\right.$, pyrazole C-4). ${ }^{15} \mathrm{~N}-\mathrm{NMR}\left(\mathrm{DMSO}-d_{6}\right): \delta 194.4$ (pyrazole N-1), 262.1 (pyrazole N-2). ${ }^{1} \mathrm{H}-\mathrm{NMR}\left(\mathrm{C}_{6} \mathrm{D}_{6}\right): \delta 12.69$ (br s, 1H, OH), 7.33 (m, 2H, Ph H-2,6), 7.06 (m, 2H, Ph H-3,5), 6.97 (d, ${ }^{3} \mathrm{~J}=2.6 \mathrm{~Hz}, 1 \mathrm{H}$, pyrazole H-5), 6.86 (m, 1H, Ph H-4), 5.76 (d, ${ }^{3} \mathrm{~J}=2.6 \mathrm{~Hz}$, $1 \mathrm{H}$, pyrazole H-4). ${ }^{13} \mathrm{C}-\mathrm{NMR}\left(\mathrm{C}_{6} \mathrm{D}_{6}\right): \delta 165.1\left({ }^{2} \mathrm{~J}(\mathrm{C} 3, \mathrm{H} 4)=2.2 \mathrm{~Hz},{ }^{3} \mathrm{~J}(\mathrm{C} 3, \mathrm{H} 5)=10.4 \mathrm{~Hz}\right.$, pyrazole C-3), 139.9 (Ph C-1), 129.7 (Ph C-3,5), $129.3\left({ }^{1} \mathrm{~J}(\mathrm{C} 5, \mathrm{H} 5)=187.1 \mathrm{~Hz},{ }^{2} \mathrm{~J}(\mathrm{C} 5, \mathrm{H} 4)=8.5 \mathrm{~Hz}\right.$, pyrazole C-5), $125.8(\mathrm{Ph}$ $\mathrm{C}-4), 118.8(\mathrm{Ph} \mathrm{C}-2,6), 94.6\left({ }^{1} J(\mathrm{C} 4, \mathrm{H} 4)=179.8 \mathrm{~Hz},{ }^{2} J(\mathrm{C} 4, \mathrm{H} 5)=7.7 \mathrm{~Hz}\right.$, pyrazole C-4). ${ }^{15} \mathrm{~N}-\mathrm{NMR}\left(\mathrm{C}_{6} \mathrm{D}_{6}\right)$ : $\delta 191.7$ (pyrazole N-1), 246.1 (pyrazole N-2). ${ }^{1} \mathrm{H}-\mathrm{NMR}\left(\mathrm{CD}_{3} \mathrm{OD}\right): \delta 7.89\left({ }^{3} \mathrm{~J}=2.6 \mathrm{~Hz}, 1 \mathrm{H}\right.$, pyrazole H-5), 7.57 (m, 2H, Ph H-2,6), 7.38 (m, 2H, Ph H-3,5), 7.17 (m, 1H, Ph H-4), 5.82 (d, ${ }^{3} \mathrm{~J}=2.6 \mathrm{~Hz}, 1 \mathrm{H}$, pyrazole $\mathrm{H}-4) .{ }^{13} \mathrm{C}-\mathrm{NMR}\left(\mathrm{CD}_{3} \mathrm{OD}\right): \delta 164.5\left({ }^{2} \mathrm{~J}(\mathrm{C} 3, \mathrm{H} 4)=2.6 \mathrm{~Hz},{ }^{3} \mathrm{~J}(\mathrm{C} 3, \mathrm{H} 5)=10.2 \mathrm{~Hz}\right.$, pyrazole C-3), $141.3(\mathrm{Ph}$ C-1), $130.4(\mathrm{Ph} \mathrm{C}-3,5), 129.7\left({ }^{1} J(\mathrm{C} 5, \mathrm{H} 5)=187.6 \mathrm{~Hz},{ }^{2} J(\mathrm{C} 5, \mathrm{H} 4)=8.6 \mathrm{~Hz}\right.$, pyrazole C-5), $126.3(\mathrm{Ph} \mathrm{C}-4)$, $118.8(\mathrm{Ph} \mathrm{C}-2,6), 94.8\left({ }^{1} \mathrm{~J}(\mathrm{C} 4, \mathrm{H} 4)=178.6 \mathrm{~Hz},{ }^{2} \mathrm{~J}(\mathrm{C} 4, \mathrm{H} 5)=7.9 \mathrm{~Hz}\right.$, pyrazole C-4). ${ }^{15} \mathrm{~N}-\mathrm{NMR}\left(\mathrm{CD}_{3} \mathrm{OD}\right)$ : $\delta 193.8$ (pyrazole N-1), 256.3 (pyrazole N-2). ${ }^{13}$ C-SSNMR: $\delta 164.9$ (pyrazole C-3), 140.1 (Ph C-1), 132.9, 
131.6 (pyrazole C-5), 131.0, 127.1, 118.8, 115.5, 95.9 (pyrazole C-4). ${ }^{1} \mathrm{H}-S S N M R: \delta 11.2$ (pyrazole OH), 7.1, 6.9, 6.9, 6.7, 5.8, 5.4 (pyrazole H-5), 5.1 (pyrazole H-4). ${ }^{13} \mathrm{C} /{ }^{1} \mathrm{H}-H E T C O R-S S N M R: ~ \delta ~ 132.9 / 6.9$, 131.6/5.4, 131.0/7.1, 127.1/6.7, 118.8/6.9, 115.5/5.8, 95.9/5.1 (pyrazole C-4). ${ }^{15} \mathrm{~N}-S S N M R: ~ \delta 243.1$ (pyrazole N-2), 192.6 (pyrazole N-1).

3-Methoxy-1-phenyl-1H-pyrazole (2) [29]. ${ }^{1} \mathrm{H}-\mathrm{NMR}\left(\mathrm{CDCl}_{3}\right): \delta 7.72\left(\mathrm{~d},{ }^{3} \mathrm{~J}=2.6 \mathrm{~Hz}, 1 \mathrm{H}\right.$, pyrazole H-5), 7.61 (m, 2H, Ph H-2,6), 7.40 (m, 2H, Ph H-3,5), 7.19 (m, 1H, Ph H-4), 5.89 (d, ${ }^{3} \mathrm{~J}=2.6 \mathrm{~Hz}, 1 \mathrm{H}$, pyrazole H-4), 3.98 (s, $\left.3 \mathrm{H}, \mathrm{OCH}_{3}\right) .{ }^{13} \mathrm{C}-\mathrm{NMR}\left(\mathrm{CDCl}_{3}\right): \delta 165.0\left({ }^{2} \mathrm{~J}(\mathrm{C} 3, \mathrm{H} 4)=2.0 \mathrm{~Hz},{ }^{3} \mathrm{~J}(\mathrm{C} 3, \mathrm{H} 5)=10.2 \mathrm{~Hz},{ }^{3} \mathrm{~J}(\mathrm{C} 3, \mathrm{OMe})=4.0 \mathrm{~Hz}\right.$, pyrazole C-3), 140.2 (Ph C-1), $129.3(\mathrm{Ph} \mathrm{C}-3,5), 127.7\left({ }^{1} J(\mathrm{C} 5, \mathrm{H} 5)=186.2 \mathrm{~Hz},{ }^{2} J(\mathrm{C} 5, \mathrm{H} 4)=8.3 \mathrm{~Hz}\right.$, pyrazole C-5), $125.2(\mathrm{Ph} \mathrm{C}-4), 117.8(\mathrm{Ph} \mathrm{C}-2,6), 93.4\left({ }^{1} J(\mathrm{C} 4, \mathrm{H} 4)=179.2 \mathrm{~Hz},{ }^{2} J(\mathrm{C} 4, \mathrm{H} 5)=8.1 \mathrm{~Hz}\right.$, pyrazole C-4), 56.3 $\left({ }^{1} \mathrm{~J}=145.3 \mathrm{~Hz}, \mathrm{OCH}_{3}\right) .{ }^{15} \mathrm{~N}-\mathrm{NMR}\left(\mathrm{CDCl}_{3}\right): \delta 195.6$ (pyrazole N-1), 261.7 (pyrazole N-2).

2-Methyl-1-phenyl-1,2-dihydro-3H-pyrazol-3-one (3) [30]. ${ }^{1} \mathrm{H}-\mathrm{NMR}\left(\mathrm{CDCl}_{3}\right): \delta 7.44(\mathrm{~m}, 2 \mathrm{H}, \mathrm{Ph} \mathrm{H}-3,5), 7.39$ $\left(\mathrm{d},{ }^{3} \mathrm{~J}=3.6 \mathrm{~Hz}, 1 \mathrm{H}\right.$, pyrazole H-5), $7.33(\mathrm{~m}, 1 \mathrm{H}, \mathrm{Ph} \mathrm{H}-4), 7.18(\mathrm{~m}, 2 \mathrm{H}, \mathrm{Ph} \mathrm{H}-2,6), 5.59\left(\mathrm{~d},{ }^{3} \mathrm{~J}=3.6 \mathrm{~Hz}, 1 \mathrm{H}\right.$, pyrazole $\mathrm{H}-4), 3.24\left(\mathrm{~s}, 3 \mathrm{H}, \mathrm{NCH}_{3}\right) .{ }^{13} \mathrm{C}-\mathrm{NMR}\left(\mathrm{CDCl}_{3}\right): \delta 168.2$ (pyrazole C-3), $142.3\left({ }^{1} \mathrm{~J}(\mathrm{C} 5, \mathrm{H} 5)=188.5 \mathrm{~Hz}\right.$, ${ }^{2} J(\mathrm{C} 5, \mathrm{H} 4)=7.7 \mathrm{~Hz}$, pyrazole C-5), 137.8 (Ph C-1), 129.9 (Ph C-3,5), 127.8 (Ph C-4), 123.0 (Ph C-2,6), 98.1 $\left({ }^{1} J(\mathrm{C} 4, \mathrm{H} 4)=182.6 \mathrm{~Hz},{ }^{2} J(\mathrm{C} 4, \mathrm{H} 5)=6.3 \mathrm{~Hz}\right.$, pyrazole C-4 $), 30.3\left({ }^{1} \mathrm{~J}=140.7 \mathrm{~Hz}, \mathrm{NCH}_{3}\right) .{ }^{15} \mathrm{~N}-\mathrm{NMR}\left(\mathrm{CDCl}_{3}\right):$ $\delta 159.1$ (pyrazole N-1), 162.5 (pyrazole N-2).

4-Chloro-1-phenyl-1H-pyrazol-3-ol (4) [29]. ${ }^{1} \mathrm{H}-\mathrm{NMR}\left(\mathrm{CDCl}_{3}\right)$ : $\delta 11.30$ (br s, $\left.1 \mathrm{H}, \mathrm{OH}\right), 7.72(\mathrm{~s}, 1 \mathrm{H}$, pyrazole $\mathrm{H}-5), 7.47(\mathrm{~m}, 4 \mathrm{H}, \mathrm{Ph} \mathrm{H}-2,3,5,6), 7.30(\mathrm{~m}, 1 \mathrm{H}, \mathrm{Ph} \mathrm{H}-4) .{ }^{13} \mathrm{C} \mathrm{NMR}\left(\mathrm{CDCl}_{3}\right): \delta 159.2\left({ }^{3} \mathrm{~J}(\mathrm{C} 3, \mathrm{H} 5)=8.5 \mathrm{~Hz}\right.$, pyrazole C-3), 139.0 (Ph C-1), 129.8 (Ph C-3,5), $126.8\left({ }^{1} \mathrm{~J}(\mathrm{C} 5, \mathrm{H} 5)=192.3 \mathrm{~Hz}\right.$, pyrazole C-5), 126.6 (Ph C-4), $118.7(\mathrm{Ph} \mathrm{C}-2,6), 98.2\left({ }^{2} J(\mathrm{C} 4, \mathrm{H} 5)=4.3 \mathrm{~Hz}\right.$, pyrazole C-4). ${ }^{15} \mathrm{~N}-\mathrm{NMR}\left(\mathrm{CDCl}_{3}\right): \delta 187.6$ (pyrazole $\mathrm{N}-1), 246.5$ (pyrazole N-2). ${ }^{1} \mathrm{H}-\mathrm{NMR}$ (DMSO- $\left.d_{6}\right): \delta 11.02(\mathrm{~s}, 1 \mathrm{H}, \mathrm{OH}), 8.52(\mathrm{~s}, 1 \mathrm{H}$, pyrazole H-5), 7.66 (m, 2H, Ph H-2,6), 7.43 (m, 2H, Ph H-3,5), 7.20 (m, 1H, Ph H-4). ${ }^{13} \mathrm{C}-\mathrm{NMR}$ (DMSO- $d_{6}$ ): $\delta 158.1$ $\left({ }^{3} J(\mathrm{C} 3, \mathrm{H} 5)=8.5 \mathrm{~Hz}\right.$, pyrazole C-3), $139.3(\mathrm{Ph} \mathrm{C}-1), 129.4(\mathrm{Ph} \mathrm{C}-3,5), 126.4\left({ }^{1} J(\mathrm{C} 5, \mathrm{H} 5)=194.4 \mathrm{~Hz}\right.$, pyrazole C-5), $125.2(\mathrm{Ph} \mathrm{C}-4), 116.7(\mathrm{Ph} \mathrm{C}-2,6), 97.2\left({ }^{2} \mathrm{~J}(\mathrm{C} 4, \mathrm{H} 5)=4.6 \mathrm{~Hz}\right.$, pyrazole C-4). ${ }^{15} \mathrm{~N}-\mathrm{NMR}\left(\mathrm{DMSO}-d_{6}\right): \delta$ 190.0 (pyrazole N-1), 262.0 (pyrazole N-2).

4-Bromo-1-phenyl-1H-pyrazol-3-ol (5) [29]. ${ }^{1} \mathrm{H}-\mathrm{NMR}\left(\mathrm{CDCl}_{3}\right)$ : $\delta 11.33$ (br s, 1H, OH), 7.73 (s, 1H, pyrazole $\mathrm{H}-5), 7.48(\mathrm{~m}, 4 \mathrm{H}, \mathrm{Ph} \mathrm{H}-2,3,5,6), 7.30(\mathrm{~m}, 1 \mathrm{H}, \mathrm{Ph} \mathrm{H}-4) .{ }^{13} \mathrm{C}-\mathrm{NMR}\left(\mathrm{CDCl}_{3}\right)$ : $\delta 160.6$ (pyrazole C-3, $\left.{ }^{3} J(\mathrm{C} 3, \mathrm{H} 5)=8.7 \mathrm{~Hz}\right), 139.1(\mathrm{Ph} \mathrm{C}-1), 129.8(\mathrm{Ph} \mathrm{C}-3,5), 129.1\left({ }^{1} \mathrm{~J}(\mathrm{C} 5, \mathrm{H} 5)=192.5 \mathrm{~Hz}\right.$, pyrazole C-5), 126.7 $(\mathrm{Ph} \mathrm{C}-4), 118.8(\mathrm{Ph} \mathrm{C}-2,6), 82.2\left({ }^{2} \mathrm{~J}(\mathrm{C} 4, \mathrm{H} 5)=4.6 \mathrm{~Hz}\right.$, pyrazole C-4). ${ }^{15} \mathrm{~N}-\mathrm{NMR}\left(\mathrm{CDCl}_{3}\right): \delta 191.7$ (pyrazole N-1), 247.9 (pyrazole N-2). ${ }^{1} \mathrm{H}-\mathrm{NMR}$ (DMSO- $\left.d_{6}\right): \delta 10.99(\mathrm{~s}, 1 \mathrm{H}, \mathrm{OH}), 8.51(\mathrm{~s}, 1 \mathrm{H}$, pyrazole H-5), 7.67 (m, 2H, Ph H-2,6), 7.43 (m, 2H, Ph H-3,5), 7.21 (m, 1H, Ph H-4). ${ }^{13} \mathrm{C}-\mathrm{NMR}$ (DMSO- $d_{6}$ ): $\delta 159.4$ $\left({ }^{3} J(\mathrm{C} 3, \mathrm{H} 5)=8.9 \mathrm{~Hz}\right.$, pyrazole C-3), $139.3(\mathrm{Ph} \mathrm{C}-1), 129.4(\mathrm{Ph} \mathrm{C}-3,5), 128.5\left({ }^{1} \mathrm{~J}(\mathrm{C} 5, \mathrm{H} 5)=194.7 \mathrm{~Hz}\right.$, pyrazole C-5), $125.3(\mathrm{Ph} \mathrm{C}-4), 116.8(\mathrm{Ph} \mathrm{C}-2,6), 82.1\left({ }^{2} \mathrm{~J}(\mathrm{C} 4, \mathrm{H} 5)=5.2 \mathrm{~Hz}\right.$, pyrazole C-4). ${ }^{15} \mathrm{~N}-\mathrm{NMR}\left(\mathrm{DMSO}-d_{6}\right): \delta$ 193.7 (pyrazole N-1), 262.5 (pyrazole N-2).

4-Iodo-1-phenyl-1H-pyrazol-3-ol (6) [29]. ${ }^{1} \mathrm{H}-\mathrm{NMR}\left(\mathrm{CDCl}_{3}\right)$ : $\delta 11.40$ (br s, 1H, OH), $7.72(\mathrm{~s}, 1 \mathrm{H}$, pyrazole $\mathrm{H}-5), 7.48(\mathrm{~m}, 4 \mathrm{H}, \mathrm{Ph} \mathrm{H}-2,3,5,6), 7.31(\mathrm{~m}, 1 \mathrm{H}, \mathrm{Ph} \mathrm{H}-4) .{ }^{13} \mathrm{C}-\mathrm{NMR}\left(\mathrm{CDCl}_{3}\right)$ : $\delta 163.6$ (pyrazole C-3, $\left.{ }^{3} J(\mathrm{C} 3, \mathrm{H} 5)=9.1 \mathrm{~Hz}\right), 139.1(\mathrm{Ph} \mathrm{C}-1), 133.3\left({ }^{1} \mathrm{~J}(\mathrm{C} 5, \mathrm{H} 5)=192.3 \mathrm{~Hz}\right.$, pyrazole C-5), $129.8(\mathrm{Ph} \mathrm{C}-3,5), 126.7$ $(\mathrm{Ph} \mathrm{C}-4), 118.8(\mathrm{Ph} \mathrm{C}-2,6), 46.6\left({ }^{2} J(\mathrm{C} 4, \mathrm{H} 5)=5.0 \mathrm{~Hz}\right.$, pyrazole C-4). ${ }^{15} \mathrm{~N}-\mathrm{NMR}\left(\mathrm{CDCl}_{3}\right): \delta 197.2($ pyrazole $\mathrm{N}-1$ ), 248.1 (pyrazole N-2). ${ }^{1} \mathrm{H}-\mathrm{NMR}\left(\mathrm{DMSO}-d_{6}\right): \delta 10.89(\mathrm{~s}, 1 \mathrm{H}, \mathrm{OH}), 8.40(\mathrm{~s}, 1 \mathrm{H}$, pyrazole $\mathrm{H}-5)$, 7.67 (m, 2H, Ph H-2,6), 7.41 (m, 2H, Ph H-3,5), 7.18 (m, 1H, Ph H-4). ${ }^{13} \mathrm{C}-\mathrm{NMR}$ (DMSO-d $d_{6}$ ): $\delta 162.7$ $\left({ }^{3} \mathrm{~J}(\mathrm{C} 3, \mathrm{H} 5)=9.4 \mathrm{~Hz}\right.$, pyrazole C-3), $139.3(\mathrm{Ph} \mathrm{C}-1), 132.5\left({ }^{1} \mathrm{~J}(\mathrm{C} 5, \mathrm{H} 5)=193.8 \mathrm{~Hz}\right.$, pyrazole C-5), $129.4(\mathrm{Ph}$ C-3,5), $125.2(\mathrm{Ph} \mathrm{C}-4), 116.8(\mathrm{Ph} \mathrm{C}-2,6), 49.0\left({ }^{2} J(\mathrm{C} 4, \mathrm{H} 5)=6.3 \mathrm{~Hz}\right.$, pyrazole C-4). ${ }^{15} \mathrm{~N}-\mathrm{NMR}\left(\mathrm{DMSO}-d_{6}\right)$ : $\delta 199.3$ (pyrazole N-1), 263.0 (pyrazole N-2).

1-(3-Hydroxy-1-phenyl-1H-pyrazol-4-yl)ethan-1-one (7) [29,31]. ${ }^{1} \mathrm{H}-\mathrm{NMR}\left(\mathrm{CDCl}_{3}\right): \delta 9.40(\mathrm{~s}, 1 \mathrm{H}, \mathrm{OH})$, 8.13 (s, 1H, pyrazole H-5), 7.67 (m, 2H, Ph H-2,6), 7.46 (m, 2H, Ph H-3,5), 7.33 (m, 1H, Ph H-4), 2.48 $\left(\mathrm{s}, 3 \mathrm{H}, \mathrm{COCH}_{3}\right) .{ }^{13} \mathrm{C}-\mathrm{NMR}\left(\mathrm{CDCl}_{3}\right): \delta 195.7(\mathrm{C}=\mathrm{O}), 164.0\left({ }^{3} \mathrm{~J}(\mathrm{C} 3, \mathrm{H} 5)=8.7 \mathrm{~Hz}\right.$, pyrazole C-3, $), 139.0$ (Ph C-1), 129.6 (Ph C-3,5), $128.1\left({ }^{1} \mathrm{~J}(\mathrm{C} 5, \mathrm{H} 5)=188.0 \mathrm{~Hz}\right.$, pyrazole C-5), $127.4(\mathrm{Ph} \mathrm{C}-4), 119.1(\mathrm{Ph} \mathrm{C}-2,6)$, $108.4\left({ }^{2} \mathrm{~J}(\mathrm{C} 4, \mathrm{H} 5)=7.8 \mathrm{~Hz},{ }^{3} \mathrm{~J}\left(\mathrm{C} 4, \mathrm{CH}_{3}\right)=1.6 \mathrm{~Hz}\right.$, pyrazole C-4), $27.0\left({ }^{1} \mathrm{~J}=127.9 \mathrm{~Hz}, \mathrm{CH}_{3}\right) .{ }^{15} \mathrm{~N}-\mathrm{NMR}$ 
$\left(\mathrm{CDCl}_{3}\right): \delta 200.4$ (pyrazole N-1), 264.2 (pyrazole N-2). ${ }^{1} \mathrm{H}-\mathrm{NMR}$ (DMSO- $\left.d_{6}\right): \delta 11.10(\mathrm{~s}, 1 \mathrm{H}, \mathrm{OH}), 8.84$ (s, 1H, pyrazole H-5), 7.80 (m, 2H, Ph H-2,6), 7.48 (m, 2H, Ph H-3,5), 7.29 (m, 1H, Ph H-4), 2.38 (s, $\left.3 \mathrm{H}, \mathrm{COCH}_{3}\right) .{ }^{13} \mathrm{C}-\mathrm{NMR}\left(\mathrm{DMSO}-d_{6}\right): \delta 191.7(\mathrm{C}=\mathrm{O}), 161.4\left({ }^{3} \mathrm{~J}(\mathrm{C} 3, \mathrm{H} 5)=9.0 \mathrm{~Hz}\right.$, pyrazole C-3), 138.8 (Ph C-1), $131.2\left({ }^{1} J(\mathrm{C} 5, \mathrm{H} 5)=191.8 \mathrm{~Hz}\right.$, pyrazole C-5), 129.4 (Ph C-3,5), 126.4 (Ph C-4), 118.0 (Ph C-2,6), $110.8\left({ }^{2} J(\mathrm{C} 4, \mathrm{H} 5)=6.8 \mathrm{~Hz},{ }^{3} \mathrm{~J}\left(\mathrm{C} 4, \mathrm{CH}_{3}\right)=1.4 \mathrm{~Hz}\right.$, pyrazole C-4), $28.5\left({ }^{1} \mathrm{~J}=127.4 \mathrm{~Hz}, \mathrm{CH}_{3}\right) .{ }^{15} \mathrm{~N}-\mathrm{NMR}$ (DMSO- $d_{6}$ ): $\delta 198.5$ (pyrazole N-1), 263.1 (pyrazole N-2).

(3-Hydroxy-1-phenyl-1H-pyrazol-4-yl)(phenyl)methanone (8) [32]. To a stirred suspension of anhydrous aluminum chloride $(16.0 \mathrm{~g}, 0.12 \mathrm{~mol})$ in $20 \mathrm{~mL}$ of carbon disulfide, maintained at room temperature with a water bath, a slurry of 1-phenyl-1H-pyrazol-3-yl benzoate (12) $(2.64 \mathrm{~g}, 10 \mathrm{mmol})$ in $70 \mathrm{~mL}$ of carbon disulfide was added. After the addition was complete, the reaction mixture was refluxed for $8 \mathrm{~h}$. After the solvent was removed under reduced pressure the residual paste was cooled in an ice bath and a solution of $13.3 \mathrm{~mL}$ of $6 \mathrm{~N}$ hydrochloric acid in $33 \mathrm{~mL}$ of ice water was added slowly under stirring to decompose the aluminum chloride salts, then the mixture was allowed to stand overnight. The solid was filtered off, washed with water, dried and recrystallized from $\mathrm{EtOH}$ to afford $818 \mathrm{mg}$ (31\%) of 8, m.p. ${ }^{138-140}{ }^{\circ} \mathrm{C}(\mathrm{EtOH}) .{ }^{1} \mathrm{H}-\mathrm{NMR}\left(\mathrm{CDCl}_{3}\right): \delta 9.91(\mathrm{~s}, 1 \mathrm{H}, \mathrm{OH}), 8.17(\mathrm{~s}, 1 \mathrm{H}$, pyrazole $\mathrm{H}-5), 7.89$ (m, 2H, CPh H-2,6), 7.69 (m, 2H, NPh H-2,6), 7.63 (m, 1H, CPh H-4), 7.54 (m, 2H, CPh $\mathrm{H}-3,5), 7.45$ (m, 2H, NPh H-3,5), 7.32 (m, 1H, NPh H-4). ${ }^{13} \mathrm{C}-\mathrm{NMR}\left(\mathrm{CDCl}_{3}\right)$ : $\delta 191.7(\mathrm{C}=\mathrm{O}), 165.6$ $\left({ }^{3} \mathrm{~J}(\mathrm{C} 3, \mathrm{H} 5)=8.8 \mathrm{~Hz}\right.$, pyrazole C-3), $139.0(\mathrm{NPh} \mathrm{C}-1), 138.0(\mathrm{CPh} \mathrm{C}-1), 132.8(\mathrm{CPh} \mathrm{C}-4), 129.5(\mathrm{NPh}$ $\mathrm{C}-3,5), 129.1\left(\left({ }^{1} J(\mathrm{C} 5, \mathrm{H} 5)=189.6 \mathrm{~Hz}\right.\right.$, pyrazole C-5), $128.9(\mathrm{CPh} \mathrm{C}-3,5), 128.2(\mathrm{CPh} \mathrm{C}-2,6), 127.4(\mathrm{NPh}$ C-4), $119.2(\mathrm{NPh} \mathrm{C}-2,6), 106.9\left({ }^{2} \mathrm{~J}(\mathrm{C} 4, \mathrm{H} 5)=8.0 \mathrm{~Hz}\right.$, pyrazole C-4). ${ }^{15} \mathrm{~N}-\mathrm{NMR}\left(\mathrm{CDCl}_{3}\right): \delta 202.1$ (pyrazole $\mathrm{N}-1), 264.0$ (pyrazole N-2). IR (KBr): $1627(\mathrm{C}=\mathrm{O}) \mathrm{cm}^{-1}$. MS m/z (\%): $265\left([\mathrm{M}+\mathrm{H}]^{+}, 100\right)$. Anal. Calcd. for $\mathrm{C}_{16} \mathrm{H}_{12} \mathrm{~N}_{2} \mathrm{O}_{2}$ : C, 72.72; $\mathrm{H}, 4.58 ; \mathrm{N}, 10.60$. Found: $\mathrm{C}, 73.01 ; \mathrm{H}, 4.65 ; \mathrm{N}, 10.27$.

4-Bromo-3-methoxy-1-phenyl-1H-pyrazole (9). The synthesis and the ${ }^{1} \mathrm{H}$ and ${ }^{13} \mathrm{C}-\mathrm{NMR}$ spectra of 9 are described in lit. [26]. ${ }^{15} \mathrm{~N}-\mathrm{NMR}\left(\mathrm{CDCl}_{3}\right): \delta 194.7$ (pyrazole N-1), 262.6 (pyrazole N-2).

1-(3-Methoxy-1-phenyl-1H-pyrazol-4-yl)ethan-1-one (10) [29]. ${ }^{1} \mathrm{H}-\mathrm{NMR}\left(\mathrm{CDCl}_{3}\right)$ : $\delta 8.25(\mathrm{~s}, 1 \mathrm{H}$, pyrazole H-5), 7.63 (m, 2H, Ph H-2,6), 7.44 (m, 2H, Ph H-3,5), 7.28 (m, 1H, Ph H-4), 4.08 (s, 3H, $\left.\mathrm{OCH}_{3}\right), 2.46$ $\left(\mathrm{s}, 3 \mathrm{H}, \mathrm{COCH}_{3}\right) \cdot{ }^{13} \mathrm{C}-\mathrm{NMR}\left(\mathrm{CDCl}_{3}\right): \delta 192.3(\mathrm{C}=\mathrm{O}), 162.6\left({ }^{3} \mathrm{~J}(\mathrm{C} 3, \mathrm{H} 5)=8.8 \mathrm{~Hz},{ }^{3} \mathrm{~J}\left(\mathrm{C}_{3}, \mathrm{OCH}_{3}\right)=3.8 \mathrm{~Hz}\right.$, pyrazole C-3), $139.1(\mathrm{Ph} \mathrm{C}-1), 130.4\left({ }^{1} \mathrm{~J}(\mathrm{C} 5, \mathrm{H} 5)=189.4 \mathrm{~Hz}\right.$, pyrazole $\left.\mathrm{C}-5\right), 129.5(\mathrm{Ph} \mathrm{C}-3,5), 126.8(\mathrm{Ph}$ C-4), $118.5(\mathrm{Ph} \mathrm{C}-2,6), 111.6\left({ }^{2} \mathrm{~J}(\mathrm{C} 4, \mathrm{H} 5)=6.6 \mathrm{~Hz},{ }^{3} \mathrm{~J}\left(\mathrm{C}_{4}, \mathrm{CH}_{3}\right)=1.4 \mathrm{~Hz}\right.$, pyrazole C-4), $56.6\left({ }^{1} \mathrm{~J}=146.5 \mathrm{~Hz}\right.$, $\left.\mathrm{OCH}_{3}\right), 29.1\left({ }^{1} \mathrm{~J}=127.8 \mathrm{~Hz}, \mathrm{CH}_{3}\right) .{ }^{15} \mathrm{~N}-\mathrm{NMR}\left(\mathrm{CDCl}_{3}\right): \delta 200.0$ (pyrazole N-1), 262.9 (pyrazole $\left.\mathrm{N}-2\right)$.

1-Phenyl-1H-pyrazol-3-yl acetate (11) [29]. ${ }^{1} \mathrm{H}-\mathrm{NMR}\left(\mathrm{CDCl}_{3}\right): \delta 7.83\left(\mathrm{~d},{ }^{3} \mathrm{~J}=2.5 \mathrm{~Hz}, 1 \mathrm{H}\right.$, pyrazole H-5), 7.62 (m, 2H, Ph H-2,6), 7.42 (m, 2H, Ph H-3,5), 7.26 (m, 1H, Ph H-4), 6.36 (d, ${ }^{3} \mathrm{~J}=2.5 \mathrm{~Hz}, 1 \mathrm{H}$, pyrazole H-4), $2.32\left(\mathrm{~s}, 3 \mathrm{H}, \mathrm{COCH}_{3}\right) \cdot{ }^{13} \mathrm{C}-\mathrm{NMR}\left(\mathrm{CDCl}_{3}\right): \delta 167.9\left({ }^{2} \mathrm{~J}\left(\mathrm{CO} ; \mathrm{CH}_{3}\right)=7.0 \mathrm{~Hz}, \mathrm{C}=\mathrm{O}\right), 156.4\left({ }^{2} \mathrm{~J}(\mathrm{C} 3, \mathrm{H} 4)=1.2 \mathrm{~Hz}\right.$, ${ }^{3} \mathrm{~J}(\mathrm{C} 3, \mathrm{H} 5)=10.9 \mathrm{~Hz}$, pyrazole C-3), $139.6(\mathrm{Ph} \mathrm{C}-1), 129.4(\mathrm{Ph} \mathrm{C}-3,5), 127.7\left({ }^{1} \mathrm{~J}(\mathrm{C} 5, \mathrm{H} 5)=188.5 \mathrm{~Hz}\right.$, ${ }^{2} \mathrm{~J}(\mathrm{C} 5, \mathrm{H} 4)=8.5 \mathrm{~Hz}$, pyrazole C-5), $126.4(\mathrm{Ph} \mathrm{C}-4), 118.6(\mathrm{Ph} \mathrm{C}-2,6), 98.8\left({ }^{1} \mathrm{~J}(\mathrm{C} 4, \mathrm{H} 4)=189.9 \mathrm{~Hz}\right.$, ${ }^{2} J(\mathrm{C} 4, \mathrm{H} 5)=8.1 \mathrm{~Hz}$, pyrazole C-4), $20.9\left({ }^{1} J=130.3 \mathrm{~Hz}, \mathrm{CH}_{3}\right) .{ }^{15} \mathrm{~N}-\mathrm{NMR}\left(\mathrm{CDCl}_{3}\right): \delta 202.9($ pyrazole $\mathrm{N}-1)$, 263.8 (pyrazole N-2).

1-Phenyl-1H-pyrazol-3-yl benzoate (12). A solution of 1 (480 mg, $3 \mathrm{mmol})$, benzoyl chloride (436 mg, $3.1 \mathrm{mmol})$ and pyridine $(0.1 \mathrm{~mL})$ in toluene $(4 \mathrm{~mL})$ was heated at $100{ }^{\circ} \mathrm{C}$ for 30 minutes. Then the reaction mixture was poured into water $(10 \mathrm{~mL})$, the precipitate was filtered off, washed with water and recrystallized from 50\% aqueous ethanol to afford $490 \mathrm{mg}(62 \%)$ of $\mathbf{1 1}$ as colourless crystals, m.p. 59-60 ${ }^{\circ} \mathrm{C}$. IR (KBr): $1745(\mathrm{C}=\mathrm{O}) \mathrm{cm}^{-1} .{ }^{1} \mathrm{H}-\mathrm{NMR}\left(\mathrm{CDCl}_{3}\right): 88.26(\mathrm{~m}, 2 \mathrm{H}, \mathrm{CPh} \mathrm{H}-2,6), 7.90\left(\mathrm{~d},{ }^{3} \mathrm{~J}=2.6 \mathrm{~Hz}\right.$, 1H, pyrazole H-5), 7.67 (m, 2H, NPh H-2,6), $7.64(\mathrm{~m}, 1 \mathrm{H}, \mathrm{CPh} \mathrm{H}-4), 7.51(\mathrm{~m}, 2 \mathrm{H}, \mathrm{CPh} \mathrm{H}-3,5), 7.45$ (m, 2H, NPh H-3,5), $7.28(\mathrm{~m}, 1 \mathrm{H}, \mathrm{NPh} \mathrm{H}-4), 6.52\left(\mathrm{~d},{ }^{3} \mathrm{~J}=2.6 \mathrm{~Hz}, 1 \mathrm{H}\right.$, pyrazole $\left.\mathrm{H}-4\right) .{ }^{13} \mathrm{C}-\mathrm{NMR}\left(\mathrm{CDCl}_{3}\right): \delta 163.8$ $(\mathrm{C}=\mathrm{O}), 156.7\left({ }^{2} \mathrm{~J}(\mathrm{C} 3, \mathrm{H} 4)=1.6 \mathrm{~Hz},{ }^{3} \mathrm{~J}(\mathrm{C} 3, \mathrm{H} 5)=11.1 \mathrm{~Hz}\right.$, pyrazole C-3), $139.7(\mathrm{NPh} \mathrm{C}-1), 133.8(\mathrm{CPh} \mathrm{C}-4)$, 130.4 (CPh C-2,6), 129.4 (NPh C-3,5), 128.8 (CPh C-1), 128.6 (CPh C-3,5), $127.8\left({ }^{1} \mathrm{~J}(\mathrm{C} 5, \mathrm{H} 5)=188.5 \mathrm{~Hz}\right.$, ${ }^{2} J(\mathrm{C} 5, \mathrm{H} 4)=8.6 \mathrm{~Hz}$, pyrazole C-5), $126.5(\mathrm{NPh} \mathrm{C}-4), 118.7(\mathrm{NPh} \mathrm{C}-2,6), 99.1\left({ }^{1} \mathrm{~J}(\mathrm{C} 4, \mathrm{H} 4)=184.1 \mathrm{~Hz}\right.$, ${ }^{2} \mathrm{~J}(\mathrm{C} 4, \mathrm{H} 5)=8.0 \mathrm{~Hz}$, pyrazole C-4). ${ }^{15} \mathrm{~N}-\mathrm{NMR}\left(\mathrm{CDCl}_{3}\right): \delta 203.2$ (pyrazole N-1), 277.7 (pyrazole N-2). 
MS $m / z(\%): 265\left([\mathrm{M}+\mathrm{H}]^{+}, 100\right)$. Anal. Calcd. for $\mathrm{C}_{16} \mathrm{H}_{12} \mathrm{~N}_{2} \mathrm{O}_{2}: \mathrm{C}, 72.72 ; \mathrm{H}, 4.58 ; \mathrm{N}, 10.60$. Found: $\mathrm{C}$, 72.93; H, 4.45; N, 10.34 .

1-Phenyl-1H-pyrazol-3-yl thiophene-2-carboxylate (13). A solution of $\mathbf{1}(3.20 \mathrm{~g}, 20 \mathrm{mmol})$ and 2-thiophenecarbonyl chloride $(2.93 \mathrm{~g}, 20 \mathrm{mmol})$ in dry toluene $(25 \mathrm{~mL})$ was refluxed for $3.5 \mathrm{~h}$. The reaction mixture was poured into water $(40 \mathrm{~mL})$, the phases were separated and the aqueous phase was extracted with toluene $(2 \times 15 \mathrm{~mL})$. The combined organic phases were dried $\left(\mathrm{Na}_{2} \mathrm{SO}_{4}\right)$ and, after filtration, evaporated under reduced pressure. The residue was recrystallized from $\mathrm{EtOH}-\mathrm{H}_{2} \mathrm{O}$ to afford $3.54 \mathrm{~g}$ (65\%) of 13 as almost colorless crystals, m.p. $62-63{ }^{\circ} \mathrm{C}$. IR $(\mathrm{KBr}): 1736(\mathrm{C}=\mathrm{O}) \mathrm{cm}^{-1} .{ }^{1} \mathrm{H}-\mathrm{NMR}\left(\mathrm{CDCl}_{3}\right)$ : $\delta 8.02\left(\mathrm{dd},{ }^{3} \mathrm{~J}(\mathrm{H} 3, \mathrm{H} 4)=3.8 \mathrm{~Hz},{ }^{4} \mathrm{~J}(\mathrm{H} 3, \mathrm{H} 5)=1.1 \mathrm{~Hz}, 1 \mathrm{H}, \mathrm{Th} \mathrm{H}-3\right), 7.88\left(\mathrm{~d},{ }^{3} \mathrm{~J}=2.6 \mathrm{~Hz}, 1 \mathrm{H}\right.$, pyrazole $\mathrm{H}-5), 7.67\left(\mathrm{dd},{ }^{3} \mathrm{~J}(\mathrm{H} 5, \mathrm{H} 4)=4.9 \mathrm{~Hz},{ }^{4} J(\mathrm{H} 5, \mathrm{H} 3)=1.3 \mathrm{~Hz}, 1 \mathrm{H}, \mathrm{Th} \mathrm{H}-5\right), 7.65(\mathrm{~m}, 2 \mathrm{H}, \mathrm{Ph} \mathrm{H}-2,6), 7.43$ (m, 2H, Ph H-3,5), 7.27 (m, 1H, Ph H-4), $7.16\left(\mathrm{dd},{ }^{3} \mathrm{~J}(\mathrm{H} 4, \mathrm{H} 3)=3.8 \mathrm{~Hz},{ }^{3} \mathrm{~J}(\mathrm{H} 4, \mathrm{H} 5)=4.9 \mathrm{~Hz}, 1 \mathrm{H}, \mathrm{Th}\right.$ $\mathrm{H}-4), 6.49\left(\mathrm{~d},{ }^{3} \mathrm{~J}=2.6 \mathrm{~Hz}\right.$, pyrazole H-4). ${ }^{13} \mathrm{C}-\mathrm{NMR}\left(\mathrm{CDCl}_{3}\right): \delta 159.1(\mathrm{C}=\mathrm{O}), 156.2\left({ }^{2} \mathrm{~J}(\mathrm{C} 3, \mathrm{H} 4)=1.5 \mathrm{~Hz}\right.$, ${ }^{3} \mathrm{~J}(\mathrm{C} 3, \mathrm{H} 5)=11.1 \mathrm{~Hz}$, pyrazole C-3), $139.6(\mathrm{NPh} \mathrm{C}-1), 135.1\left({ }^{1} \mathrm{~J}=171.3 \mathrm{~Hz},{ }^{2} \mathrm{~J}=5.6 \mathrm{~Hz},{ }^{3} \mathrm{~J}=9.2 \mathrm{~Hz}, \mathrm{Th}\right.$ C-3), $134.1\left({ }^{1} J=185.7 \mathrm{~Hz},{ }^{2} J=7.3 \mathrm{~Hz},{ }^{3} J=11.2 \mathrm{~Hz}\right.$, Th C-5 $), 131.9\left({ }^{2} J=5.8 \mathrm{~Hz},{ }^{3} J(C 2, H 4)=9.7 \mathrm{~Hz}\right.$, ${ }^{3} \mathrm{~J}(\mathrm{C} 3, \mathrm{H} 5)=5.8 \mathrm{~Hz}$, Th C-2), $129.3(\mathrm{PhC}-3,5), 128.0\left({ }^{1} \mathrm{~J}=170.6 \mathrm{~Hz},{ }^{2} \mathrm{~J}(\mathrm{C} 4, \mathrm{H} 3)=5.0 \mathrm{~Hz},{ }^{2} \mathrm{~J}(\mathrm{C} 4, \mathrm{H} 5)=4.0 \mathrm{~Hz}\right.$, Th C-4), $127.7\left({ }^{1} J(\mathrm{C} 5, \mathrm{H} 5)=188.6 \mathrm{~Hz},{ }^{2} J(\mathrm{C} 5, \mathrm{H} 4)=8.5 \mathrm{~Hz}\right.$, pyrazole C-5), $126.4(\mathrm{Ph} \mathrm{C}-4), 118.6(\mathrm{Ph} \mathrm{C}-2,6)$, $99.0\left({ }^{1} \mathrm{~J}(\mathrm{C} 4, \mathrm{H} 4)=184.4 \mathrm{~Hz},{ }^{2} \mathrm{~J}(\mathrm{C} 4, \mathrm{H} 5)=8.1 \mathrm{~Hz}\right.$, pyrazole C-4). ${ }^{15} \mathrm{~N}-\mathrm{NMR}\left(\mathrm{CDCl}_{3}\right): \delta 203.3($ pyrazole $\mathrm{N}-1$ ), 277.8 (pyrazole N-2). MS $m / z$ (\%): $271\left([\mathrm{M}+\mathrm{H}]^{+}, 100\right)$. Anal. Calcd. for $\mathrm{C}_{14} \mathrm{H}_{10} \mathrm{~N}_{2} \mathrm{O}_{2} \mathrm{~S}: \mathrm{C}, 62.21$; H, 3.73; N, 10.36. Found: C, 62.51; H, 4.01; N, 9.97.

4-Bromo-1-(4-bromophenyl)-1H-pyrazol-3-ol (14). The synthesis and spectral data of $\mathbf{1 4}$ are given in [33].

Preparation of compounds 15 and 16. 4-Bromo-1-(4-bromophenyl)-1H-pyrazol-3-ol 14 (1.0 g, 3.15 mmol) was dissolved in DMF $(20 \mathrm{~mL})$ and potassium hydroxide $(204 \mathrm{mg}, 3.65 \mathrm{mmol})$ was added to the solution. The mixture was stirred for $15 \mathrm{~min}$, then allyl bromide (442 mg, $3.65 \mathrm{mmol}$ ) was added, and stirring was continued for $30 \mathrm{~min}$. The reaction mixture was poured into water $(40 \mathrm{~mL})$ and extracted with ether $(3 \times 30 \mathrm{~mL})$. The combined organic extracts were dried over anhydrous sodium sulphate, the solvent was then evaporated under reduced pressure, and the residue subjected to column chromatography (silica gel, eluent: hexane-ethyl acetate 3:1) to yield $824 \mathrm{mg}(73 \%)$ of compound 15 $\left(\mathrm{R}_{f} 0.68\right)$ and $58 \mathrm{mg}(5 \%)$ of compound $\mathbf{1 6}\left(\mathrm{R}_{f} 0.37\right)$ as oily substances.

4-Bromo-1-(4-bromophenyl)-3-(prop-2-en-1-yloxy)-1H-pyrazole (15). ${ }^{1} \mathrm{H}-\mathrm{NMR}\left(\mathrm{CDCl}_{3}\right)$ : $\delta 7.74(\mathrm{~s}, 1 \mathrm{H}$, pyrazole H-5), $7.51(\mathrm{~m}, 2 \mathrm{H}, \mathrm{Ph} \mathrm{H}-2,6), 7.42(\mathrm{~m}, 2 \mathrm{H}, \mathrm{Ph} \mathrm{H}-3,5), 6.12\left(\mathrm{~m}, 1 \mathrm{H}, \mathrm{C} \underline{\mathrm{H}}=\mathrm{CH}_{2}\right), 5.46(\mathrm{dd}$, ${ }^{2} J=1.4 \mathrm{~Hz},{ }^{3} \mathrm{~J}=17.2 \mathrm{~Hz}, 1 \mathrm{H}, \mathrm{CH}=\mathrm{CH}_{2}$ (trans)), $5.31\left({ }^{2} \mathrm{~J}=1.4 \mathrm{~Hz},{ }^{3} \mathrm{~J}=10.5 \mathrm{~Hz}, 1 \mathrm{H}, \underline{\mathrm{CH}}=\mathrm{C}_{2}\right.$ (cis)), 4.83 $\left(\mathrm{m}, \mathrm{OCH}_{2}\right) .{ }^{13} \mathrm{C}-\mathrm{NMR}\left(\mathrm{CDCl}_{3}\right): \delta 160.5\left({ }^{3} \mathrm{~J}(\mathrm{C} 3, \mathrm{H} 5)=5.1 \mathrm{~Hz},{ }^{3} \mathrm{~J}\left(\mathrm{C}_{3}, \mathrm{OCH}_{2}\right)=2.8 \mathrm{~Hz}\right.$, pyrazole C-3 $), 138.6$ (Ph C-1), $132.6\left(\mathrm{CH}=\mathrm{CH}_{2}\right), 132.4(\mathrm{Ph} \mathrm{C}-3,5), 127.5\left({ }^{1} \mathrm{~J}=191.9 \mathrm{~Hz}\right.$, pyrazole C-5), $118.9(\mathrm{Ph} \mathrm{C}-2,6), 118.6$ $(\mathrm{Ph} \mathrm{C}-4), 118.4\left(\mathrm{CH}=\mathrm{CH}_{2}\right), 83.1\left({ }^{2} \mathrm{~J}(\mathrm{C} 4, \mathrm{H} 5)=5.1 \mathrm{~Hz}\right.$, pyrazole C-4 $), 70.2\left(\mathrm{OCH}_{2}\right) .{ }^{15} \mathrm{~N}-\mathrm{NMR}\left(\mathrm{CDCl}_{3}\right)$ : $\delta 192.2$ (pyrazole N-1), 262.6 (pyrazole N-2). MS m/z (\%): 361/359/357 ([M + H $\left.]^{+}, 49 / 100 / 51\right)$. Anal. Calcd. for $\mathrm{C}_{12} \mathrm{H}_{10} \mathrm{Br}_{2} \mathrm{~N}_{2} \mathrm{O}: \mathrm{C}, 40.26 ; \mathrm{H}, 2.82 ; \mathrm{N}, 7.82$. Found: C, 40.40; H, 2.90; N 7.69.

4-Bromo-1-(4-bromophenyl)-2-(prop-2-en-1-yl)-1,2-dihydro-3H-pyrazol-3-one (16). ${ }^{1} \mathrm{H}-\mathrm{NMR}\left(\mathrm{CDCl}_{3}\right): \delta 7.58$ (m, 2H, Ph H-3,5), 7.52 (s, 1H, pyrazole H-5), 7.08 (m, 2H, Ph H-2,6), $5.63\left(\mathrm{~m}, 1 \mathrm{H}, \mathrm{C} \underline{\mathrm{H}}=\mathrm{CH}_{2}\right), 5.09$ (m, $1 \mathrm{H}, \mathrm{CH}=\mathrm{CH}_{2}$ (cis)), $4.91\left(\mathrm{~m}, 1 \mathrm{H}, \mathrm{CH}=\mathrm{CH}_{2}\right.$ (trans)), $4.34\left(\mathrm{~m}, \mathrm{NCH}_{2}\right) .{ }^{13} \mathrm{C}-\mathrm{NMR}\left(\mathrm{CDCl}_{3}\right): \delta 164.2$ (pyrazole C-3), $142.5\left({ }^{1} \mathrm{~J}=193.9 \mathrm{~Hz}\right.$, pyrazole C-5), $136.7(\mathrm{Ph} \mathrm{C}-1), 133.2(\mathrm{Ph} \mathrm{C}-3,5), 130.9\left(\underline{\mathrm{CH}}=\mathrm{CH}_{2}\right)$, 125.2 (Ph C-2,6), $122.2(\mathrm{Ph} \mathrm{C}-4), 119.0\left(\mathrm{CH}=\mathrm{CH}_{2}\right), 90.1\left({ }^{2} \mathrm{~J}(\mathrm{C} 4, \mathrm{H} 5)=3.0 \mathrm{~Hz}\right.$, pyrazole C-4), $46.3\left(\mathrm{NCH}_{2}\right)$. ${ }^{15} \mathrm{~N}-\mathrm{NMR}\left(\mathrm{CDCl}_{3}\right): \delta 150.8$ (pyrazole N-1), 170.8 (pyrazole N-2). IR (KBr): $1659(\mathrm{C}=\mathrm{O}) \mathrm{cm}^{-1}$. MS m/z (\%): 361/359/357 ([M+ H $\left.]^{+}, 49 / 100 / 51\right)$. Anal. Calcd. for $\mathrm{C}_{12} \mathrm{H}_{10} \mathrm{Br}_{2} \mathrm{~N}_{2} \mathrm{O}: \mathrm{C}, 40.26 ; \mathrm{H}, 2.82 ; \mathrm{N}, 7.82$. Found: $\mathrm{C}, 40.51 ; \mathrm{H}, 3.15 ; \mathrm{N}, 8.13$.

1-Methyl-1H-pyrazol-3-ol (17) [34]. ${ }^{1} \mathrm{H}-\mathrm{NMR}\left(\mathrm{CDCl}_{3}\right): \delta 12.00(\mathrm{~s}, 1 \mathrm{H}, \mathrm{OH}), 7.07\left(\mathrm{~d},{ }^{3} J=2.4 \mathrm{~Hz}, 1 \mathrm{H}\right.$, pyrazole $\mathrm{H}-5), 5.56\left(\mathrm{~d},{ }^{3} \mathrm{~J}=2.4 \mathrm{~Hz}, 1 \mathrm{H}\right.$, pyrazole $\left.\mathrm{H}-4\right), 3.69\left(\mathrm{~s}, 3 \mathrm{H}, \mathrm{NCH}_{3}\right) .{ }^{13} \mathrm{C}-\mathrm{NMR}\left(\mathrm{CDCl}_{3}\right): \delta 162.6$ (pyrazole C-3), 131.8 (pyrazole C-5), 90.6 (pyrazole C-4), $38.3\left(\mathrm{NCH}_{3}\right) .{ }^{15} \mathrm{~N}-\mathrm{NMR}\left(\mathrm{CDCl}_{3}\right): \delta 172.1$ (pyrazole N-1), 252.0 (pyrazole N-2). ${ }^{1} \mathrm{H}-\mathrm{NMR}\left(\mathrm{DMSO}_{-} d_{6}\right): \delta 9.53(\mathrm{~s}, 1 \mathrm{H}, \mathrm{OH}), 7.30\left(\mathrm{~d},{ }^{3} \mathrm{~J}=2.2 \mathrm{~Hz}\right.$, 
$1 \mathrm{H}$, pyrazole $\mathrm{H}-5), 5.39\left(\mathrm{~d},{ }^{3} \mathrm{~J}=2.2 \mathrm{~Hz}, 1 \mathrm{H}\right.$, pyrazole $\left.\mathrm{H}-4\right), 3.58\left(\mathrm{~s}, 3 \mathrm{H}, \mathrm{NCH}_{3}\right) .{ }^{13} \mathrm{C}-\mathrm{NMR}\left(\mathrm{DMSO}-d_{6}\right)$ : $\delta 160.9$ (pyrazole C-3), 131.3 (pyrazole C-5), 89.8 (pyrazole C-4), $38.2\left(\mathrm{NCH}_{3}\right) .{ }^{15} \mathrm{~N}-\mathrm{NMR}\left(\mathrm{DMSO}-d_{6}\right): \delta$ 177.2 (pyrazole N-1), 271.8 (pyrazole N-2). ${ }^{1} \mathrm{H}-\mathrm{NMR}\left(\mathrm{C}_{6} \mathrm{D}_{6}\right): \delta 12.56(\mathrm{~s}, 1 \mathrm{H}, \mathrm{OH}), 6.28\left(\mathrm{~d},{ }^{3} \mathrm{~J}=2.4 \mathrm{~Hz}\right.$, $1 \mathrm{H}$, pyrazole $\mathrm{H}-5), 5.62\left(\mathrm{~d},{ }^{3} \mathrm{~J}=2.4 \mathrm{~Hz}, 1 \mathrm{H}\right.$, pyrazole $\left.\mathrm{H}-4\right), 2.90\left(\mathrm{~s}, 3 \mathrm{H}, \mathrm{NCH}_{3}\right) .{ }^{13} \mathrm{C}-\mathrm{NMR}\left(\mathrm{C}_{6} \mathrm{D}_{6}\right): \delta 163.7$ (pyrazole C-3), 131.5 (pyrazole C-5), 90.8 (pyrazole C-4), $37.4\left(\mathrm{NCH}_{3}\right) .{ }^{15} \mathrm{~N}-\mathrm{NMR}\left(\mathrm{C}_{6} \mathrm{D}_{6}\right): \delta 171.8$ (pyrazole N-1), 252.7 (pyrazole N-2).

1-Benzyl-1H-pyrazol-3-ol (18) [34]. ${ }^{1} \mathrm{H}-\mathrm{NMR}\left(\mathrm{CDCl}_{3}\right): \delta 11.30$ (s, 1H, OH), 7.33 (m, 3H, Ph H-3,4,5), $7.25(\mathrm{~m}, 2 \mathrm{H}, \mathrm{Ph} \mathrm{H}-2,6), 7.11\left(\mathrm{~d},{ }^{3} \mathrm{~J}=2.5 \mathrm{~Hz}, 1 \mathrm{H}\right.$, pyrazole $\left.\mathrm{H}-5\right), 5.62\left(\mathrm{~d},{ }^{3} \mathrm{~J}=2.5 \mathrm{~Hz}, 1 \mathrm{H}\right.$, pyrazole $\left.\mathrm{H}-4\right)$, $5.07\left(\mathrm{~s}, 2 \mathrm{H}, \mathrm{NCH}_{2}\right) .{ }^{13} \mathrm{C}-\mathrm{NMR}\left(\mathrm{CDCl}_{3}\right): \delta 162.6\left({ }^{2} \mathrm{~J}(\mathrm{C} 3, \mathrm{H} 4)=2.2 \mathrm{~Hz},{ }^{3} \mathrm{~J}(\mathrm{C} 3, \mathrm{H} 5)=10.1 \mathrm{~Hz}\right.$, pyrazole C-3), $136.0(\mathrm{Ph} \mathrm{C}-1), 130.9\left({ }^{1} J(\mathrm{C} 5, \mathrm{H} 5)=185.8 \mathrm{~Hz},{ }^{2} \mathrm{~J}(\mathrm{C} 5, \mathrm{H} 4)=8.2 \mathrm{~Hz},{ }^{3} J\left(\mathrm{C}^{2}, \mathrm{NCH}_{2}\right)=3.2 \mathrm{~Hz}\right.$, pyrazole C-5), 128.8 (Ph C-3,5), 128.1 (Ph C-4), 127.8 (Ph C-2,6), $91.3\left({ }^{1} \mathrm{~J}(\mathrm{C} 4, \mathrm{H} 4)=178.9 \mathrm{~Hz},{ }^{2} J(\mathrm{C} 4, \mathrm{H} 5)=8.0 \mathrm{~Hz}\right.$, pyrazole C-4), $55.4\left({ }^{1} J=139.3 \mathrm{~Hz}, \mathrm{NCH}_{2}\right) .{ }^{15} \mathrm{~N}-\mathrm{NMR}\left(\mathrm{CDCl}_{3}\right): \delta 183.5$ (pyrazole N-1), 251.2 (pyrazole $\mathrm{N}-2) .{ }^{1} \mathrm{H}-\mathrm{NMR}$ (DMSO- $\left.d_{6}\right): \delta 9.63(\mathrm{~s}, 1 \mathrm{H}, \mathrm{OH}), 7.50\left(\mathrm{~d},{ }^{3} \mathrm{~J}=2.3 \mathrm{~Hz}, 1 \mathrm{H}\right.$, pyrazole $\left.\mathrm{H}-5\right), 7.32(\mathrm{~m}, 2 \mathrm{H}$, Ph H-3,5), 7.27 (m, 1H, Ph H-4), 7.19 (m, 2H, Ph H-2,6), 5.47 (d, ${ }^{3} J=2.3 \mathrm{~Hz}, 1 \mathrm{H}$, pyrazole H-4), 5.05 $\left(\mathrm{s}, 2 \mathrm{H}, \mathrm{NCH}_{2}\right) .{ }^{13} \mathrm{C}-\mathrm{NMR}\left(\mathrm{DMSO}-d_{6}\right): \delta 161.3\left({ }^{2} J(\mathrm{C} 3, \mathrm{H} 4)=2.5 \mathrm{~Hz},{ }^{3} \mathrm{~J}(\mathrm{C} 3, \mathrm{H} 5)=10.1 \mathrm{~Hz}\right.$, pyrazole C-3), $138.0(\mathrm{Ph} \mathrm{C}-1), 131.2\left({ }^{1} \mathrm{~J}(\mathrm{C} 5, \mathrm{H} 5)=185.8 \mathrm{~Hz},{ }^{2} \mathrm{~J}(\mathrm{C} 5, \mathrm{H} 4)=8.5 \mathrm{~Hz},{ }^{3} J\left(\mathrm{C} 5, \mathrm{NCH}_{2}\right)=3.1 \mathrm{~Hz}\right.$, pyrazole C-5), 128.3 (Ph C-3,5), 127.4 (Ph C-2,6), 127.3 (Ph C-4), $90.3\left({ }^{1} J(\mathrm{C} 4, \mathrm{H} 4)=176.2 \mathrm{~Hz},{ }^{2} \mathrm{~J}(\mathrm{C} 4, \mathrm{H} 5)=8.6 \mathrm{~Hz}\right.$, pyrazole C-4), $54,5\left({ }^{1} J=139.2 \mathrm{~Hz}, \mathrm{NCH}_{2}\right) .{ }^{15} \mathrm{~N}-\mathrm{NMR}$ (DMSO- $\left.d_{6}\right): \delta 187.9$ (pyrazole N-1), 269.9 (pyrazole N-2). ${ }^{1} \mathrm{H}-\mathrm{NMR}\left(\mathrm{C}_{6} \mathrm{D}_{6}\right): \delta 12.40(\mathrm{~s}, 1 \mathrm{H}, \mathrm{OH}), 6.93-7.05(\mathrm{~m}, 5 \mathrm{H}, \mathrm{Ph} \mathrm{H}), 6.45\left(\mathrm{~d},{ }^{3} \mathrm{~J}=2.4 \mathrm{~Hz}, 1 \mathrm{H}\right.$, pyrazole $\mathrm{H}-5), 5.63\left(\mathrm{~d},{ }^{3} \mathrm{~J}=2.4 \mathrm{~Hz}, 1 \mathrm{H}\right.$, pyrazole $\left.\mathrm{H}-4\right), 4.51\left(\mathrm{~s}, 2 \mathrm{H}, \mathrm{NCH}_{2}\right) \cdot{ }^{13} \mathrm{C}-\mathrm{NMR}\left(\mathrm{C}_{6} \mathrm{D}_{6}\right): \delta 164.2$ $\left({ }^{2} \mathrm{~J}(\mathrm{C} 3, \mathrm{H} 4)=2.2 \mathrm{~Hz},{ }^{3} \mathrm{~J}(\mathrm{C} 3, \mathrm{H} 5)=10.1 \mathrm{~Hz}\right.$, pyrazole C-3 $), 137.0(\mathrm{Ph} \mathrm{C}-1), 131.4\left({ }^{1} \mathrm{~J}(\mathrm{C} 5, \mathrm{H} 5)=185.0 \mathrm{~Hz}\right.$, ${ }^{2} \mathrm{~J}(\mathrm{C} 5, \mathrm{H} 4)=8.2 \mathrm{~Hz},{ }^{3} \mathrm{~J}\left(\mathrm{C} 5, \mathrm{NCH}_{2}\right)=3.1 \mathrm{~Hz}$, pyrazole C-5), $129.2(\mathrm{Ph} \mathrm{C}-3,5), 128.4(\mathrm{Ph} \mathrm{C}-4), 128.4(\mathrm{Ph}$ $\mathrm{C}-2,6), 92.0\left({ }^{1} J(\mathrm{C} 4, \mathrm{H} 4)=178.2 \mathrm{~Hz},{ }^{2} J(\mathrm{C} 4, \mathrm{H} 5)=8.1 \mathrm{~Hz}\right.$, pyrazole C-4), $55.6\left({ }^{1} J=139.2 \mathrm{~Hz}, \mathrm{NCH}_{2}\right)$. ${ }^{15} \mathrm{~N}-\mathrm{NMR}\left(\mathrm{C}_{6} \mathrm{D}_{6}\right)$ : $\delta 183.3$ (pyrazole $\left.\mathrm{N}-1\right), 251.9$ (pyrazole $\mathrm{N}-2$ ).

\subsection{X-ray Crystal Structure Analysis}

The X-ray intensity data was measured on a Bruker X8 APEXII equipped with multilayer monochromators, with a Mo K/a INCOATEC micro focus sealed tube, and a Kryoflex II cooling device. The structure was solved by direct methods and refined by full-matrix least-squares techniques. Non-hydrogen atoms were refined with anisotropic displacement parameters. The hydrogen located at $\mathrm{O} 1$ was refined without any restraints or constraints. All other hydrogen atoms were inserted at calculated positions and refined with a riding model. The following software was used: Frame integration, Bruker SAINT software package [35] using a narrow-frame algorithm, Absorption correction, SADABS [36], structure solution, SHELXL-2013 [37], refinement, SHELXL-2013 [37], OLEX2 [38], SHELXLE [39], molecular diagrams, OLEX2 [38]. Experimental data and CCDC-Code [40] can be found in Table 1. Crystal data, data collection parameters, and structure refinement details are given in Tables 2 and 3. Molecular structures in "Ortep View" are displayed in Figures 2 and 9. Bond length details are given in Table 4.

Table 1. Experimental parameter and CCDC-Code.

\begin{tabular}{ccccccccc}
\hline Sample & Machine & Source & Temp. & Detector Distance & Time/Frame & \#Frames & Frame Width & CCDC \\
\hline & Bruker & & {$[\mathrm{K}]$} & {$[\mathrm{mm}]$} & {$[\mathrm{s}]$} & & {$\left[{ }^{\circ}\right]$} & \\
$\mathbf{1}$ & X8 & Mo & 100 & 35 & 25 & 3739 & 0.5 & 1586020 \\
\hline
\end{tabular}




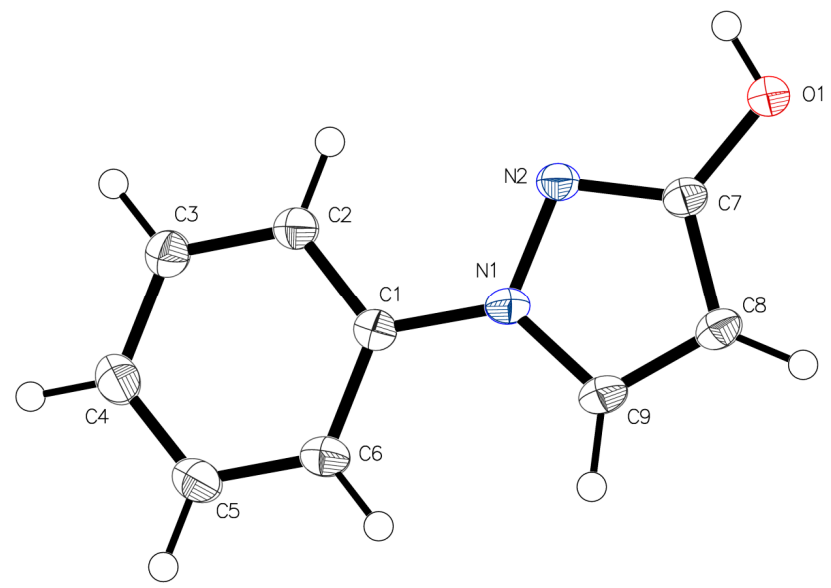

Figure 9. Asymmetric unit of 1, drawn with 50\% displacement ellipsoids.

Table 2. Sample and crystal data of $\mathbf{1}$.

\begin{tabular}{|c|c|c|c|}
\hline Chemical Formula & $\mathrm{C}_{9} \mathrm{H}_{8} \mathrm{~N}_{2} \mathrm{O}$ & Crystal System & Orthorhombic \\
\hline Formula weight (g/mol) & 160.17 & Space group & $\mathrm{Pbca}$ \\
\hline Temperature (K) & 100 & $\mathrm{Z}$ & 8 \\
\hline Measurement method & $\Phi$ and $\omega$ scans & Volume $\left(\AA^{3}\right)$ & 1509.4(3) \\
\hline Radiation (Wavelength (Å)) & $\operatorname{MoK} \alpha(\lambda=0.71073)$ & Unit cell dimensions $(\AA)$ and $\left(^{\circ}\right)$ & $13.6517(13)$ \\
\hline Crystal size $\left(\mathrm{mm}^{3}\right)$ & $0.22 \times 0.12 \times 0.04$ & & $6.3663(6)$ \\
\hline Crystal habit & clear colourless plate & & $17.3673(17)$ \\
\hline Density (calculated) $\left(\mathrm{g} / \mathrm{cm}^{3}\right)$ & 1.41 & Absorption coefficient/(mm $\left.{ }^{-1}\right)$ & 0.096 \\
\hline Abs. correction $T_{\min }$ & 0.703 & Abs. correction $T_{\max }$ & 0.746 \\
\hline Abs. correction type & multi-scan & $F(000)\left(e^{-}\right)$ & 672 \\
\hline
\end{tabular}

Table 3. Data collection and structure refinement of $\mathbf{1}$.

\begin{tabular}{|c|c|c|c|}
\hline Index ranges & $\begin{array}{l}-19 \leq \mathrm{h} \leq 19,-9 \leq \\
\mathrm{k} \leq 8,-24 \leq 1 \leq 24\end{array}$ & $\begin{array}{l}\text { Theta range for data } \\
\text { collection }\left({ }^{\circ}\right)\end{array}$ & 4.69 to 60.5 \\
\hline Reflections number & 62937 & Data/restraints/parame & $2243 / 0 / 113$ \\
\hline Refinement method & Least squares & \multirow{2}{*}{ Final $\mathrm{R}$ indices } & $\mathrm{R} 1=0.0473, \mathrm{wR} 2=0.1264$ \\
\hline Function minimized & $\Sigma \mathrm{w}\left(\mathrm{F}_{\mathrm{o}}^{2}-\mathrm{F}_{\mathrm{c}}^{2}\right)^{2}$ & & $\mathrm{R} 1=0.0407, \mathrm{wR} 2=0.1202$ \\
\hline Goodness-of-fit on $\mathrm{F}^{2}$ & 1.085 & \multirow{2}{*}{ Weighting scheme } & $\mathrm{w}=1 /\left(\sigma^{2}\left(\mathrm{~F}_{\mathrm{o}}{ }^{2}\right)+(0.0657 \mathrm{P})^{2}+0.6517 \mathrm{P}\right)$ \\
\hline $\begin{array}{c}\text { Largest diff. peak and } \\
\text { hole }\left(\mathrm{e}^{-3}\right)\end{array}$ & $0.39 /-0.21$ & & where $\mathrm{P}=\left(\mathrm{F}_{\mathrm{o}}^{2}+2 \mathrm{~F}_{\mathrm{c}}^{2}\right) / 3$ \\
\hline
\end{tabular}

The difference electron density map (Figure 2) gives very detailed information about the position of the searched hydrogen atom. The electron density distance of the latter to $\mathrm{N}^{\prime}$ - -as displayed in Figure 2-excludes the possibility of a single bond to the nitrogen for the concerning hydrogen atom. Additionally, its distance to $\mathrm{O} 1$ for the electron density proves the position of the hydrogen is located at the oxygen. The free refinement of the hydrogen position without using any restraints or constraints clears all doubts about the non-tautomeric geometry of the molecule in the solid state. Furthermore, at least two very close molecules [41,42] were already measured and interpreted in the same way. In these samples also two identical hydrogen bonds build up molecule pairs because of symmetry reasons.

Pyrazole is well known in crystallography and its different bonds are well characterized by the Handbook of Chemistry and Physics [43]. Table 4 compares the results from compound 1 with corresponding bonds in pyrazole. In detail the double bond N2=C7 in $\mathbf{1}$ is with $1.329 \AA$ identical to the unweighted mean of the table value for pyrazoles $\mathrm{N} 2=\mathrm{C} 3$ from the Handbook of Chemistry and Physics. The single bond C7-O1 with $1.339 \AA$ is also in strong correlation to expected values like in enols, $1.333 \AA$, given. Double bond values like in lactams, $1.240 \AA$, and benzoquinones, $1.222 \AA$ are in 
contrast to the measured $1.339 \AA$ too small. Finally we proved the position of the searched hydrogen at $\mathrm{O} 1$ and we can exclude the $\mathrm{NH}$ form in the solid crystalline state.

Table 4. Proof of the bond length for the position of $\mathrm{H}$ at O1 [43].

\begin{tabular}{|c|c|c|c|c|c|c|c|}
\hline \multicolumn{6}{|c|}{ Bond Lengths in Crystalline Organic Compounds } & \multicolumn{2}{|l|}{ Compound 1} \\
\hline & d & m & $\sigma$ & $\mathrm{q}_{1}$ & $q_{u}$ & & \\
\hline in pyrazole: (N1-N2) & 1.366 & 1.366 & 0.019 & 1.350 & 1.375 & N1 N2 single bond & 1.376 \\
\hline in pyrazole: $(\mathrm{N} 2=\mathrm{C} 3)$ & 1.329 & 1.331 & 0.014 & 1.315 & 1.339 & N1 C7 double bond & 1.329 \\
\hline in pyrazole: (N1-C5) & 1.357 & 1.359 & 0.012 & 1.347 & 1.365 & N1 C9 single bond & 1.354 \\
\hline in enols: $\mathrm{C}=\mathrm{C}-\mathrm{OH}$ & 1.333 & 1.331 & 0.017 & 1.324 & 1.342 & C7 O1 single bond & 1.339 \\
\hline in phenols: $\mathrm{C}_{\text {aromatic }}-\mathrm{OH}$ & 1.362 & 1.364 & 0.015 & 1.353 & 1.373 & & \\
\hline in lactams: $(\mathrm{C}=\mathrm{O})$ & 1.240 & 1.241 & 0.003 & 1.237 & 1.243 & & \\
\hline in benzoquinones: $(\mathrm{C}=\mathrm{O})$ & 1.222 & 1.220 & 0.013 & 1.211 & 1.231 & & \\
\hline
\end{tabular}

Acknowledgments: The solid state NMR experiments were performed at the Upper Austrian-South Bohemian Research Infrastructure Center "RERI-uasb" in Linz, co-financed by the European Union in the context of the project EFRE RU2-EU-124/100-2010 (ETC Austria-Czech Republic 2007-2013, project M00146).

Author Contributions: E.A., S.K., A.M., and V.M. accomplished the syntheses; M.B. recorded the solid state NMR spectra; A.R. performed the X-ray structure analysis; A.B. and W.H. conducted the liquid state NMR studies; and W.H. and A.Š. wrote the paper.

Conflicts of Interest: The authors declare no conflict of interest.

\section{References}

1. Elguero, J.; Goya, P.; Jagerovic, N.; Silva, A.M.S. Pyrazoles as drugs: Facts and fantasies. In Targets in Heterocyclic Systems; Attanasi, O.A., Spinelli, D., Eds.; Royal Society of Chemistry: Cambridge, UK, 2002; Volume 6, pp. 52-98.

2. Perez-Fernandez, R.; Goya, P.; Elguero, J. A review of recent progress (2002-2012) on the biological activities of pyrazoles. ARKIVOC 2014, ii, 233-293.

3. Schmidt, A.; Dreger, A. Recent advances in the chemistry of pyrazoles. Properties, biological activities, and syntheses. Curr. Org. Synth. 2011, 15, 1423-1463. [CrossRef]

4. Fustero, S.; Sánchez-Roselló, M.; Barrio, P.; Simón-Fuentes, A. From 2000 to Mid-2010: A fruitful Decade for the Synthesis of Pyrazoles. Chem. Rev. 2011, 111, 6984-7034. [CrossRef] [PubMed]

5. Ansari, A.; Ali, A.; Asif, M.; Shamsuzzaman. Biologically active pyrazole derivatives. New J. Chem. 2017, 41, 16-41. [CrossRef]

6. Lamberth, C. Pyrazole Chemistry in Crop Protection. Heterocycles 2007, 71, 1467-1502. [CrossRef]

7. Holzer, W.; Claramunt, R.M.; Lopez, C.; Alkorta, I.; Elguero, J. A study in desmotropy. Solid State Nucl. Magn. Reson. 2008, 34, 68-76. [CrossRef] [PubMed]

8. Elguero, J.; Marzin, C.; Katritzky, A.R.; Linda, P. The Tautomerism of Heterocycles; Academic Press: New York, NY, USA, 1976; ISBN 012020651X.

9. Minkin, V.I.; Garnovskii, A.D.; Elguero, J.; Katritzky, A.R.; Denisko, O.V. The tautomerism of heterocycles: Five-membered rings with two or more heteroatoms. Adv. Heterocycl. Chem. 2000, 76, 157-323.

10. Holzer, W.; Mereiter, K.; Plagens, B. 4-Acyl-5-methyl-2-phenylpyrazolones: NMR and X-ray structure investigations. Heterocycles 1999, 50, 799-818. [CrossRef]

11. Holzer, W.; Hallak, L. Synthesis and NMR spectroscopic investigations with 3-amino-, 3-hydroxy-, and 3-methoxy-4-acyl-1-phenyl-2-pyrazolin-5-ones. Heterocycles 2004, 63, 1311-1334. [CrossRef]

12. Holzer, W.; Kautsch, C.; Laggner, C.; Claramunt, R.M.; Pérez-Torralba, M.; Alkorta, I.; Elguero, J. On the Tautomerism of Pyrazolones: The Geminal ${ }^{2} J[$ Pyrazole C-4,H-3(5)] Spin Coupling Constant as a Diagnostic Tool. Tetrahedron 2004, 60, 6791-6805. [CrossRef]

13. Guillou, S.; Janin, Y.L. 5-Iodo-3-Ethoxypyrazoles: An Entry Point to New Chemical Entities. Chem. Eur. J. 2010, 16, 4669-4677. [CrossRef] [PubMed] 
14. Arbačiauskienè, E.; Laukaitytè, V.; Holzer, W.; Šačkus, A. Metal-free intramolecular alkyne-azide cycloaddition leading to the novel pyrazolo[4,3-f][1,2,3]triazolo[5,1-c][1,4]oxazepine ring system. Eur. J. Org. Chem. 2015, 5663-5670.

15. Li, Y.; Liu, R.; Yan, Z.; Zhang, X.; Zhu, H. Synthesis, Crystal Structure and Fungicidal Activities of New Type Oxazolidinone-Based Strobilurin Analogues. Bull. Korean Chem. Soc. 2010, 31, 3341-3347. [CrossRef]

16. Li, Y.; Liu, Y.-Y.; Chen, N.-Q.; Lue, K.-Z.; Xiong, X.-H.; Li, J. One-Pot Regioselective Synthesis of Novel Oximino Ester-Containing 1-Aryl-4-chloro-3-oxypyrazoles as Potential Fungicides. Helv. Chim. Acta 2014, 97, 1269-1282. [CrossRef]

17. Liu, Y.; Li, Y.; Chen, N.; Lv, K.; Zhou, C.; Xiong, X.; Li, F. Synthesis and Fungicidal Activity of Novel Chloro-Containing 1-Aryl-3-oxypyrazoles with an Oximino Ester or Oximino Amide Moiety. Molecules 2014, 19, 8140-8150. [CrossRef] [PubMed]

18. Elguero, J.; Katritzky, A.R.; Denisko, O.V. Prototropic Tautomerism of Heterocycles: Heteroaromatic Tautomerism-General Overview and Methodology. In Advances in Heterocyclic Chemistry; Academic Press: Cambridge, MA, USA, 2000; Volume 76, pp. 1-84.

19. Sanz, D.; Claramunt, R.M.; Alkorta, I.; Elguero, J. The use of chemical shifts vs. coupling constants for studying tautomerism: A combined experimental and theoretical approach. Struct. Chem. 2007, 18, 703-708. [CrossRef]

20. Katritzky, A.R.; Karelson, M.; Harris, P.A. Prototropic tautomerism of heteroaromatic compounds. Heterocycles 1991, 32, 329-369. [CrossRef]

21. Von Philipsborn, W.; Müller, R. ${ }^{15}$ N-NMR Spectroscopy-New Methods and Application. Angew. Chem. Int. Ed. Engl. 1986, 25, 383-413. [CrossRef]

22. Gil, V.M.S.; von Philipsborn, W. Effect of electron lone-pairs on nuclear spin-spin coupling constants. Magn. Reson. Chem. 1989, 27, 409-430. [CrossRef]

23. Berger, S.; Braun, S.; Kalinowski, H.-O. NMR-Spektroskopie von Nichtmetallen: ${ }^{15}$ N-NMR-Spektroskopie; Thieme: Stuttgart, Germany; New York, NY, USA, 1992; Volume 2, pp. 42-43, ISBN 3-13-769101-X.

24. Begtrup, M. Hydrogen-1 and carbon-13-NMR spectra of phenyl-substituted azole derivatives. II. Conformational study. Acta Chem. Scand. B 1974, 28, 61-77. [CrossRef]

25. Carrillo, J.R.; Cossio, F.P.; Diaz-Ortiz, A.; Gomez-Escalonilla, M.J.; de la Hoz, A.; Lecea, B.; Moreno, A.; Prieto, P. A complete model for the prediction of ${ }^{1} \mathrm{H}$ - and ${ }^{13} \mathrm{C}-\mathrm{NMR}$ chemical shifts and torsional angles in phenyl-substituted pyrazoles. Tetrahedron 2001, 57, 4179-4187. [CrossRef]

26. Kleizienė, N.; Arbačiauskienè, E.; Holzer, W.; Šačkus, A. 4-Bromo-3-methoxy-1-phenyl-1H-pyrazole. Molbank 2009, M639. [CrossRef]

27. Kalinowski, H.-O.; Berger, S.; Braun, S. ${ }^{13}$ C-NMR Spektroskopie; Thieme: Stuttgart, Germany; New York, NY, USA, 1984; p. 194.

28. Arbačiauskienė, E.; Vilkauskaite, G.; Eller, G.A.; Holzer, W.; Sackus, A. Pd-Catalyzed Cross-Coupling Reactions of Halogenated 1-Phenylpyrazol-3-ols and Related Triflates. Tetrahedron 2009, 65, 7817-7824. [CrossRef]

29. O'Brien, D.F.; Gates, J.W., Jr. Some Reactions of 3-Hydroxy-1-phenylpyrazole. J. Org. Chem. 1966, 31, 1538-1542. [CrossRef]

30. Begtrup, M. Reactions between azolium salts and nucleophilic reagents. IX. Cine-substitution of pyrazolium salts. Acta Chem. Scand. 1973, 27, 2051-2074. [CrossRef]

31. Arbačiauskienè, E.; Martynaitis, V.; Krikštolaitytė, S.; Holzer, W.; Šačkus, A. Synthesis of 3-substituted 1-phenyl-1H-pyrazole-4-carbaldehydes and the corresponding ethanones by Pd-catalysed cross-coupling reactions. ARKIVOC 2011, xi, 1-21.

32. Mikhaleva, M.A.; Mamaev, V.P. Interaction of 3-hydroxy- and 4-hydroxypyrazoles with benzylidenebisurea. Izv. Sib. Otd. Akad. Nauk SSSR Seriya Khimicheskikh Nauk 1969, 6, 93-98.

33. Nedzelskyte, E.; Martynaitis, V.; Šačkus, A.; Eller, G.A.; Holzer, W. Synthesis of Mono- and Dibromo-Derivatives of 1-Phenylpyrazol-3-ol. Molbank 2007, M551. [CrossRef]

34. Sucrow, W.; Mentzel, C.; Slopianka, M. Enehydrazines. 9. 1-Alkyl-3-hydroxypyrazoles from hydrazones or hydrazines. Chem. Ber. 1974, 107, 1318-1328. [CrossRef]

35. Bruker SAINT, V7.68A; Copyright (C) 2005-2018 Bruker AXS: Madison, WI, USA, 2012.

36. Sheldrick, G.M. SHELXS; University of Göttingen: Göttingen, Germany, 1996.

37. Sheldrick, G.M. A short history of SHELX. Acta Cryst. 2008, A64, 112-122. [CrossRef] [PubMed] 
38. Dolomanov, O.V.; Bourhis, L.J.; Gildea, R.J.; Howard, J.A.K.; Puschmann, H. OLEX2: A complete structure solution, refinement and analysis program. J. Appl. Cryst. 2009, 42, 339-341. [CrossRef]

39. Huebschle, C.B.; Sheldrick, G.M.; Dittrich, B. ShelXle: A Qt graphical user interface for SHELXL. J. Appl. Cryst. 2011, 44, 1281-1284. [CrossRef] [PubMed]

40. CCDC 1586020 Contains the Supplementary Crystallographic Data for This Paper. Available online: http:/ / www.ccdc.cam.ac.uk/conts/retrieving.html (accessed on 16 November 2017).

41. Bechtel, F.; Gaultier, J.; Hauw, C. 1-Phenyl-5-methyl-3-pyrazolone, C10H10N2O. Cryst. Struct. Commun. 1973, 2, 473-476.

42. Ren, X.-Y.; Wang, J.-G.; Li, Y.-Y. 1-(4-Chlorophenyl)-1H-pyrazol-3-ol. Acta Cryst. 2010, E66, o186. [CrossRef] [PubMed]

43. Bond Lengths in Crystalline Organic Compounds. In Handbook of Chemistry and Physics, 96th Edition 2015/16; CRC Press: Boca Raton, FL, USA, 2015; ISBN 1482260972.

Sample Availability: Samples of the compounds 1, 4 and 5 are available from the authors. 